\title{
Time Course of Object Identification: Evidence for a Global-to-Local Contingency
}

\author{
Thomas Sanocki
}

\begin{abstract}
A time course contingency is the modification of later phases of object recognition contingent upon stimulus information extracted earlier in processing. It can increase the efficiency of later processing and reduce computational burdens. This idea was instantiated within a global-to-local model and supported in 4 integration priming experiments, in which primes and target objects were presented briefly and then masked. In Experiments 1-3, global and coarse-grained common-feature primes presented early in processing facilitated discriminations between similarly shaped objects, even though they provided no discrimination-relevant information. In Experiment 4, global primes were more effective than local primes early in processing, whereas local primes were more effective than global primes late in processing.
\end{abstract}

An important goal in the study of perceptual identification is to specify the time course of the relevant processes. In this endeavor, marked success has been achieved with pleasingly simple feature accumulation models, in which features are extracted over time and continuously activate form-level representations in a passive, bottom-up manner. For example, such models account for a wide variety of data on pattern and word perception (e.g., Estes, 1978; Keren \& Baggen, 1981; Massaro \& Sanocki, in press; Oden, 1979; Sanocki, 1990, 1991b; Shibuya \& Bundesen, 1988; Townsend, 1981).

The assumption of a bottom-up flow of information can be elaborated on to begin to account for object identification (e.g., Biederman, 1987). However, the adequacy of a bottom-up approach becomes questionable when the computational burdens of identifying three-dimensional objects are considered. Objects vary in size, orientation, and details of instances. They have many features that must be bound together, and objects with moving parts can vary greatly in shape. Any object can appear under different lighting conditions or be partially occluded. There is evidence indicating that the perceptual system can be sensitive to details of instances of letters (e.g., Sanocki, 1987, 1988, 1990, 1991b) and objects (e.g., Jacoby, Baker, \& Brooks, 1989; Price \& Humphreys, 1989) and to differing object orientations (e.g., Jolicoeur, 1985; Palmer, Rosch, \& Chase, 1981). This sen-

This research was supported in part by Grant DBS-9213246 from the National Science Foundation and by a Research and Creative Scholarship Award from the University of South Florida. A report of Experiments 1-3 was presented at the 1991 Annual Meeting of the Psychonomic Society, Atlanta, Georgia.

I thank Irv Biederman, Asher Cohen, Pierre Jolicoeur, Charles Leith, Lise Paquet, Jim Pomerantz, and Devida Teller for helpful comments and Beth Chapman, Tracie Gillingham, Tammy Larson, Chris Guy, Carole Young, and the members of the Graphic Cognition Laboratory for their help in conducting the experiments.

Correspondence concerning this article should be addressed to Thomas Sanocki, Department of Psychology, BEH 339, University of South Florida, Tampa, Florida 33620-8200. Electronic mail may be sent to sanocki@figment.csee.usf.edu. sitivity may extend to differing part configurations and sufficiently different lighting conditions. If during object identification the perceptual system considered such factors for an unconstrained set of alternatives, the enormous number of combinations of stimulus features and feature-object mappings would create combinatorial explosion.

These considerations support hypotheses that are not strictly bottom-up in nature. Of interest is the possibility that computational burdens may be reduced by using early stimulus information to constrain or modify later perceptual processing (Sanocki, 1991a). Such an effect is termed a time course contingency to emphasize that later processing is modified contingent upon earlier information. In this article, I develop the general idea of a time course contingency and report on tests of an instantiation of the idea in terms of a global-to-local processing model.

\section{Time Course Contingencies Versus Feature Accumulation}

The primary theoretical distinction drawn herein is between time course contingencies and feature accumulation models. ${ }^{1}$

\section{General Feature Model}

Feature accumulation models shall be represented by the general feature model, in which feature information accumulates continuously and in parallel, cascading upward to activate form-level interpretations. As in many feature models (e.g., Keren \& Baggen, 1981; Massaro \& Friedman, 1990;

\footnotetext{
${ }^{1}$ Biederman's (1987) model is a significant extension of feature accumulation models. However, it has not been developed in enough detail to provide time course predictions, and Biederman acknowledged that it may be necessary to add some assumptions that are not bottom-up to the model. This model (as well as the more recent Hummel \& Biederman, 1992, model) is considered further in the General Discussion section.
} 
Oden, 1979; Sanocki, 1990; Townsend \& Ashby, 1982), the probability of a given identification depends on the evidence for that identification, relative to the evidence for all other relevant identifications (Luce choice rule; Luce, 1959).

In this model, the accumulation of feature information is entirely passive, although some types of features may be extracted earlier than other features. In particular, a number of investigators have proposed that global features are extracted before local features and thus preponderate early in processing (e.g., Eriksen, O'Hara, \& Eriksen, 1982; Lupker, 1979; Townsend, Hu, \& Kadlec, 1988). However, unlike the contingency hypothesis, later processes are not modified in the feature model; global features may preponderate early in processing, but they do not affect the efficiency of later local processing.

\section{Contingency Hypothesis}

Information also accumulates over time in this hypothesis. However, in contrast to bottom-up models, it is assumed that later perceptual processing is modified on the basis of early information (Sanocki, 1991a). For example, early feature information may be used to delimit a class of candidate interpretations or activate initial representations. Subsequently, more detailed feature information will first be interpreted with respect to representations established in early processing. Of course, if the features are inconsistent with early interpretations, the early interpretations would have to be changed. However, when early and late information are consistent, the constraints on the interpretation of later information allow the system to process late-arriving details without the immense computational burdens of an unconstrained set of interpretations. This contrasts with the general feature model, in which incoming information has continual, unconstrained access to higher level interpretations or representations. (The computational burdens arising from unconstrained access are not obvious in many feature models, but this may be because such models have typically been applied to relatively simple stimulus domains.)

The contingency hypothesis is a general idea that could be instantiated within a variety of possible models. To motivate the present experiments, I instantiated the contingency hypothesis in terms of a global-to-local processing model. This type of model was chosen because global-to-local processing makes computational sense and because there is evidence to be reviewed that supports the idea. Furthermore, there are some interesting implications of global-to-local processing that have not yet been tested. The present global-to-local model is not very detailed, but it does make distinct predictions. The general idea of time course contingencies is considered further in the General Discussion section.

In the global-to-local model, processing is conceptualized as the construction of a structural network over time, by processes that are capacity demanding but obligatory. The main function of early processing is to establish a global representation of the stimulus. The representation consists of relatively large-scale, coarse structures and relations. Later processing uses more detailed and local stimulus information to define the network at smaller scales. Later local processes are not independent of early global processes because the availability of global structures increases the efficiency of interpreting local information. For example, global structures may provide a rough reference frame within which local information can be interrelated and localized, or the structures may attract resources that can be devoted to processing local information that is consistent with them.

There are important similarities and differences between the present proposals and existing literatures. The general strategy of using early analyses to modify later processing has become almost standard in the computer vision literature. In psychology, the idea has also existed for some time (e.g., Navon, 1977; Neisser, 1967, 1976; Norman \& Bobrow, 1976; Palmer, 1975). However, the idea typically has been instantiated in terms of fairly strong mechanisms, such as top-down effects of prior knowledge and interactions between distinct processing levels (e.g., Henderson, 1977; Neisser, 1967; Rumelhart, 1977). For example, one hypothesis was that exposure to a scene's name, semantic-level identification, expedites visual-level identification of objects within the scene (e.g., Biederman, Teitelbaum, \& Mezzanotte, 1983). Most of the data are inconsistent with strong top-down hypotheses (e.g., Biederman et al., 1983, Johnston, 1978), and few current models include strong topdown mechanisms. However, this leaves a largely unexplored middle ground between the strictly bottom-up and strong top-down approaches.

A number of the preceding ideas have been suggested or supported in prior work. The idea that pattern and object recognition are capacity demanding but obligatory has been supported by recent studies (e.g., Biederman, Blickle, Teitelbaum, Klatsky, \& Mezzanotte, 1988; Paap \& Ogden, 1981). Structural networks have been fruitfully applied to perception (e.g., Oden, 1979; Palmer, 1977; Sanocki, 1991b; Sanocki \& Oden, 1991), and the general idea of global-tolocal processing has existed for some time (e.g., Broadbent, 1977; Eriksen et al., 1982; Lupker, 1979; Navon, 1977; Townsend et al., 1988). Navon (1991) independently suggested that there may be qualitative global-to-local changes over the time course of recognition. However, as I shall explain in the next section, previous evidence does not distinguish between time course contingencies and a simple preponderance of global (or other) features early in processing.

\section{Prior Evidence Relevant to Time Course Contingencies}

There is relatively little work that addresses the idea of contingencies in perceptual identification, although the general importance of contingencies has been pointed out by several investigators (e.g., Biederman, 1972; Garner, 1985). Biederman (1972) used a classification task with tridimensional stimuli in which a cuing dimension signaled which of two other dimensions was relevant to the response. Subjects were able to use such contingencies to decrease reaction time. Garner $(1985,1987)$ used a generally similar paradigm, although the cuing dimensions in Garner's tasks did not eliminate possible responses. Garner found that when the relevant 
discrimination was difficult, processing was facilitated when location was the cuing dimension but not when color was the cuing dimension. Garner suggested that contingent cues may be helpful when they signal a channel (e.g., a location) for processing. These studies indicate that contingencies can be important in processing, but the findings were based on simple stimuli composed of a few separable dimensions. In contrast, the present emphasis is on the immediate identification of somewhat complex objects that vary along multiple dimensions, many of which may be integral or relational in nature.

In the literature on pattern identification, there are several types of evidence consistent with the general idea that the percept evolves during identification from a global, bloblike form consisting of global features to a more detailed pattern. Identification confusions for briefly presented patterns tend to be more frequent between patterns with similar shapes (e.g., Bouma, 1971; Lupker, 1979), as would be expected if global features such as shape preponderate early in processing. When same-different judgments are made with letters under conditions of speed-stress, false same responses increase in frequency, especially for visually similar but different pairs (Krueger \& Chignell, 1985). This would be expected because similar patterns would have similar global features and therefore similar percepts early in processing time. Also, Townsend and his colleagues (e.g., Townsend \& Ashby, 1982; Townsend et al., 1988) has found that the probabilities of identifying components of briefly presented patterns tend to be correlated, as might be expected if components are initially encoded within global features. Whereas this evidence supports the idea that global features preponderate early in processing, it does not indicate whether early information affects the efficiency of later processing.

In other relevant work, Humphreys and Quinlan (1988) found that reference frames established by primes facilitated later shape identification. However, facilitation may have resulted because the primes provided target-relevant information (e.g., axes), rather than because they increased the efficiency of later processing. Earhard (1990; Earhard \& Walker, 1985) has reported evidence consistent with an outside-in processing strategy in the processing of forms (see also McClelland \& Miller, 1979).

Another literature that may be relevant to the time course of identification is that on global precedence (e.g., Navon, 1977). In a typical experiment, subjects identify hierarchical forms (e.g., a large $S$ or $H$ made of smaller $S$ s or $H s$ ) at either the larger (global) or smaller (local) scale. The main finding is that, relative to responses at the smaller scale, responses at the larger scale are generally faster and suffer less from inconsistency at the smaller scale (within certain boundary conditions; e.g., see Kimchi \& Palmer, 1985; Kinchla \& Wolfe, 1979; Navon, 1977, 1981). More recently, Navon (1991) examined the time course of processing at the two scales and found precedence of the larger scale throughout the time course. Navon $(1981,1991)$ argued that the precedence effects reflect a general perceptual dominance for global features (larger forms) over local features (smaller forms) that may be caused by several mechanisms. Although the idea of global precedence early in processing is consistent with the present idea of global-to-local processing, Navon's conclusion that global precedence is constant throughout the time course of processing is inconsistent with the changes over time argued for herein. Navon's (1991) conclusions may be discounted, however, because of potential problems with terminology and interpretation (see, e.g., Miller, 1981; Pomerantz, 1983; Sanocki, 1991b). One problem is that Navon never established that the larger and smaller forms are in fact perceptual features. Although it is difficult to establish the features actually used in any given situation, Navon's paradigm is perhaps better conceptualized as comparing the priority of recognizing forms at either of two size scales (Kimchi \& Palmer, 1985). A second problem is that there is now considerable evidence that global precedence arises because larger forms are a higher priority for attentional or decisional processes, rather than because they activate mental representations earlier (e.g., Boer \& Keuss, 1982; Miller, 1981; Sanocki, 1991b). Of primary importance in the present context, however, is the fact that the local and global levels of hierarchical stimuli are strictly independent of each other. Independence between levels contrasts with natural stimuli, in which global differences are often correlated with local differences, and independence can make contingencies involving early global information irrelevant.

In sum, the literature is generally consistent with the claim that subjects can use certain simple contingencies to constrain processing and the general idea that global information is important early in processing. However, the data do not distinguish between the contingency hypothesis and a general feature model in which global features preponderate early in processing. A new paradigm in which the contingency hypothesis and the feature model make contrasting predictions in pattern identification is now described. The paradigm was extended to object identification in the present experiments.

\section{Integration Priming Paradigm}

The general idea of this paradigm is to present a shape prime briefly, followed immediately by a brief target in the same location (Sanocki, 1991a). At short durations, the prime and target are integrated (e.g., Eriksen \& Collins, 1967). In two of the experiments by Sanocki (1991a), the target set consisted of four items $(t, f, e$, and $c$ ) that formed two "similar-shape sets" as defined by outline. One prime was generated for each shape set; it contained only shape features common to the targets within the set (e.g., a cross-like prime was generated for the shape set $t$ and $f$ ). As shown in Figure 1 , each trial began with a visual warning signal, which was followed in the same location by a blank interval, a prime (on prime trials), a target, a mask, and then a forced choice. The crucial trials were those in which the primes were from the same shape set as the target (consistent prime) and the forced choice was between the two similar-shape alternatives in the set (e.g., the cross-like prime preceded $t$ or $f$, and the forced choice was between $t$ and $f$ ). Because the prime in this paradigm contains only features common to the two alternatives, it provides no discrimination-relevant information. Nevertheless, the contingency hypothesis predicts facilitation be- 


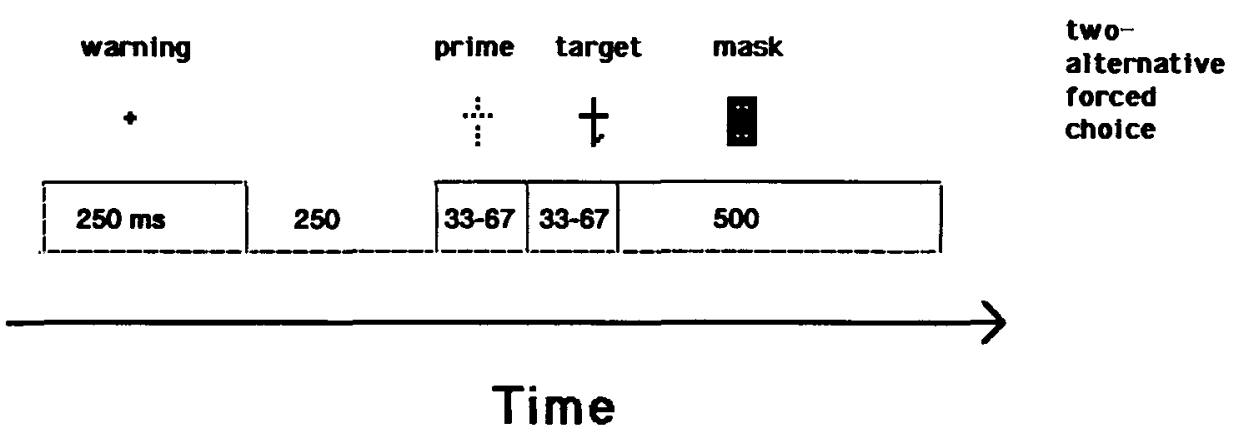

Figure 1. The time course of events on each trial in the Sanocki (1991a) experiments.

cause the prime provides early information that can increase the efficiency of later processing (e.g., by activating a global representation that is used in later processing).

In contrast, the general feature model cannot predict facilitation because the prime provides only common features. The general feature model's prediction follows from the relative goodness rule used in the aforementioned models to describe identification (Luce, 1959): The strength of one identification $\left(S_{i}\right)$ corresponds to the evidence for that identification $\left(E_{i}\right)$ relative to the sum of the evidence for all alternatives $\left(\Sigma E_{j}\right)$,

$$
S_{i}=E_{i} / \sum E_{j}
$$

In a no-prime condition, the accuracy of a similar-shape discrimination should be related to the difference in strength between the alternatives $i$ and $k$ :

$$
E_{i} / \sum E_{j}-E_{k} / \sum E_{j}=\left(E_{i}-E_{k}\right) / \sum E_{j} .
$$

A similar-shape prime would provide a fixed and equal additional amount of evidence, $P$, for each of the two relevant targets:

$$
\begin{aligned}
{\left[\left(E_{i}+P\right)-\left(E_{k}+P\right)\right] /\left(\sum E_{j}\right.} & +2 P) \\
& =\left(E_{i}-E_{k}\right) /\left(\sum E_{j}+2 P\right) .
\end{aligned}
$$

Accuracy cannot be higher in the primed condition because the numerators in Equations 2 and 3 are equal but the denominator is greater in Equation 3. This holds regardless of the globality of the features. ${ }^{2}$

In four experiments, Sanocki (1991a) found that relative to control conditions, consistent primes had facilitative effects of $5-8 \%$ on discriminations between similar-shape alternatives. The effect did not appear to depend on the type of mask (cf. Johnston \& McClelland, 1973; McClelland, 1978) because similar facilitation effects were obtained when a black mask was used (Experiment 1; the letters were black on white) and when a pattern mask was used (Experiment 2). The effect was not limited to small sets of targets that could be distinguished on the basis of a few details because in the third experiment, facilitation was obtained with a set of 12 target letters that formed four similar-shape sets. The effect also did not require highly learned representations because in the fourth experiment, novel letterlike patterns were used and, once more, facilitation was obtained. Finally, the effect did not seem to depend on the control or baseline condition used because it was obtained both with a no-prime baseline (Experiments 1, 2, and 4) and with an inconsistent-prime baseline (Experiment 3 ). In sum, a facilitation effect occurred that was consistent with the contingency hypothesis but was not predicted by the general feature model.

One other result was that, in three of Sanocki's (1991a) experiments in which there was an inconsistent-prime condition and a no-prime condition, there was no difference between these two conditions with similar-shape alternatives. Thus, an inconsistent prime did not inhibit discriminations. This result might be taken as evidence against the contingency hypotheses, because early analyses of an inconsistent prime would be assumed to provide information that is inappropriate for the target. However, given the relative simplicity of the patterns, it is possible that the effects of the prime were not strong enough to interfere with the targetappropriate processing that would begin soon after target onset. It is also possible that the "true" neutral baseline may have been underestimated slightly in those experiments (Sanocki, 1991a). Effects of inconsistent primes were examined further in the present experiments.

\footnotetext{
${ }^{2}$ In deriving the predictions, a consistent prime and target were assumed to activate only features of similar-shape items, which seems a reasonable assumption. In most cases, similar predictions would be derived if the prime and target are assumed to also affect different-shape items. To understand this, consider first the worst case for the prediction: The target activates features of differentshape items, and the prime provides evidence against differentshape items. Relative to no prime, the prime could have a positive effect within the general feature model because its negative effect on different-shape items would counteract target-based activation of those items and thereby reduce evidence in the denominator. However, because the prime also has the effect of adding evidence to the denominator (evidence for the consistent target and foil, $2 P$ ), facilitation would be predicted only if the prime's negative effect on different-shape items is greater in magnitude than its positive effect on similar-shape items (i.e., greater than $2 P$ ). This seems unlikely, because different-shape items share no actual stimulus segments with the target and therefore should not be highly activated by the target in the first place.
} 


\section{Purposes of the Present Experiments}

Three major goals motivated the present experiments. One goal of Experiments 1-3 was to attempt to extend the finding of facilitation for common-feature shape primes to the identification of (pictures of) three-dimensional objects. A second goal was to begin examining the precision of the early analyses that presumably cause facilitation: Do the early analyses produce an imprecise global representation, as in the globalto-local model? The major goal of Experiment 4 was to test a further prediction of the global-to-local model: that the relative contribution of global and local information to the eventual percept changes over the time course of recognition.

The objects in the present experiments can be termed "constructed objects." They were based on common objects, and their structures were representative of real-world objects. Because the objects were generally familiar, they were expected to be easy for subjects to encode and remember. However, particular instances were constructed in a controlled manner, as dictated by theoretical concerns. This means that the objects can provide a sensitive test tube for initial exploration of hypotheses. The objects are shown in Figure 2; the frames were used to position the objects and were never presented to subjects. The objects formed two similar-shape classes of three objects each (three houses and three vehicles). Objects within a class had generally similar overall shapes but differed on both global details (e.g., details of shape) and local details (i.e., interior features). Experiment 4 provided some validation of a distinction between global and local features in these stimuli.

\section{Experiment 1a}

In order to determine whether common-feature shape primes facilitate object identification, primes were constructed for each of the two shape classes. The primes were constructed from contours that were common to objects within a class and that conveyed the global shape (the largest scale shape) of objects in the class. The main events on each trial were the presentation of a prime, a target object, and then a forced choice. The forced choice was between the three target objects in the same shape class as the target (i.e., between the three houses or the three vehicles). The contingency hypothesis predicts that common-feature primes will produce facilitation because they provide information that can increase the efficiency of later processing.

In contrast, the general feature model cannot predict facilitation because common features do not aid in discrimination. This can be seen for the case of three-object discriminations by substituting into Equations 2 and 3 . In a neutral-prime condition, the accuracy of a similar-shape discrimination should be related to the difference in strength between the target, $i$, and the similar-shape alternatives, $k$ and $l$,

$$
\begin{array}{r}
E_{i} / \sum E_{j}-\left(E_{k} / \sum E_{j}+E_{i} / \sum E_{j}\right) \\
=\left(E_{i}-E_{k}-E_{l}\right) / \sum E_{j} .
\end{array}
$$

A common-feature prime would provide a fixed and equal additional amount of evidence, $P$, for each of the three relevant targets:

$$
\begin{aligned}
{\left[\left(E_{i}+P\right)-\left(E_{k}\right.\right.} & \left.+P)-\left(E_{l}+P\right)\right] /\left(\sum E_{j}+3 P\right) \\
& =\left(E_{i}-E_{k}-E_{l}-P\right) /\left(\sum E_{j}+3 P\right) .
\end{aligned}
$$

Accuracy cannot be higher in the primed condition because the numerator is now less in Equation 5 than in Equation 4, and the denominator is greater in Equation 5.

In the contingency hypothesis, the primes' effect is assumed to be visual in nature. An alternative possibility is that the primes produce facilitation because they provide semantic information about the class of the target. This idea was examined in Experiment $1 \mathrm{~b}$.

A second goal in Experiment la was to begin determining the precision of the early analysis that presumably causes the facilitation effect. One way to define the precision of the early analysis is in terms of its grain size-the size of regions over which information becomes averaged. Precise analyses would have a small grain size, whereas imprecise analyses would blur high-frequency details and have a relatively large grain size. In the global-to-local model, the early analyses are imprecise, and therefore fine-grained information is not necessary. The alternative hypothesis is that precise information is necessary and that the facilitative effects of primes are reduced if fine-grained information is lacking.

Grain size was varied in Experiment la by controlling the precision of the primes. The precise primes consisted of contours taken from the targets and presented in the same location as target contours. Imprecise primes were generated by duplicating the contours of the precise prime and moving them equal distances away from their original location (see Figure 3). These primes were termed echo primes. Echo primes lacked fine-grained information about the location of the target's contours. However, if echo primes were processed by a medium or large grain-size analyzer, then the contours would be averaged together, and the resultant information would be at least as effective as that provided by precise primes. The precise primes were termed nonecho primes. Two conditions of Experiment la consisted of consistent echo and nonecho primes, from the same shape class as the target. In a third condition, inconsistent nonecho primes were used (e.g., the house prime was presented before a vehicle).

In the present experiments, a prestimulus mask (as well as poststimulus mask) was used to reduce the discriminability of the objects. Also, on a given trial, the prime and target were presented at the same one of four parafoveal locations surrounding a central fixation mark. Thus, attention was attracted away from fixation to the stimulus location. In order to control for attention-attracting effects of the content primes, neutral primes were also used. Two types of neutral primes were used, in case different neutral primes yielded different baseline estimates.

\section{Method}

Stimuli and design. The target stimuli consisted of three houses and three vehicles, similar to those shown in Figure 2. The six 
HOUSES

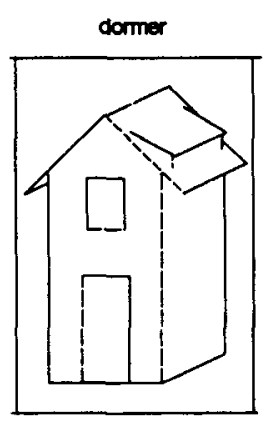

colonial

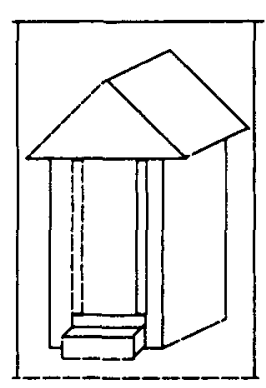

artout

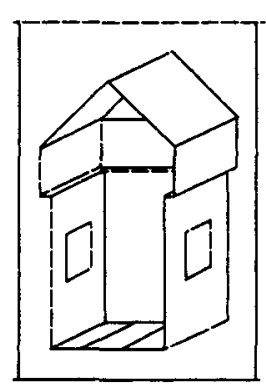

Figure 2. The six target objects used in Experiments $2-4$.

primes used are shown in Figure 3a. The four content primes consisted of common shape features of the houses or vehicles. The two neutral primes were a cross and a diamond. There were five types of prime in total: two consistent primes (echo and nonecho), one inconsistent prime (nonecho), and two neutral primes.

At the viewing distance of approximately $70 \mathrm{~cm}$, the stimuli appeared centered on one of four corners of an imaginary square, $4.3^{\circ}$ from a central fixation mark. The houses were $3.4^{\circ} \times 2.2^{\circ}$ (Height $\times$ Width), and the vehicles were $2.3^{\circ} \times 4.5^{\circ}$. The lines were approximately $1.3 \mathrm{~min}$ in width. Two of the houses differed from those in Figure 2: The dormer on the dormer stimulus was larger, and the cutout stimulus had no windows. (For all of the later experiments, these houses were changed to be like those in Figure 2 , in order to better equate overall performance levels between objects. In Experiment 1a, overall performance was near the ceiling for the dormer and relatively low for the cutout). In some of the drawings, there were small breaks in the lines produced by the drawing program used, as can be seen in Figure 2 . The particular lines that were broken depended on where the object was located on the screen.

In the echo primes, the contours were moved diagonally, approximately equal distances away from their original locations. The resulting horizontal and vertical separations were approximately 14 $\mathrm{min}$. For each of the two neutral primes, there were two versions, one for use with each object class. A given version was positioned so it would be centered with respect to the objects in its class and so its contours overlapped minimally with major contours of those objects. The neutral primes shown in Figure 3 were used with the houses.

The stimuli were displayed in black on a white background on a Macintosh SE computer, within a $9.1^{\circ} \times 13.9^{\circ}$ screen. The stimuli were preceded and followed by a mask that covered most of the screen. The mask consisted of haphazardly arranged horizontal, vertical, and oblique lines (approximately $2.6 \mathrm{~min}$ thick), with a fixation cross in the center.

Responses were made on a forced-choice display that was organized as four columns. The leftmost and rightmost columns consisted of the three houses and vehicles, respectively. In this display, the targets were reduced somewhat in size, to fit onto the screen. The two middle columns each consisted of three small rectangles positioned relatively close to the vertical center; each rectangle contained the name of the object in the corresponding position. Subjects made responses by clicking in one of these rectangles (this minimized the amount of mouse movement necessary). On a given trial, one side of the forced-choice display was obscured by a gray pattern, limiting the response choices to objects of the class on the opposite side of the screen.

Procedure. Each trial consisted of the following sequence of stimuli: the mask $(300 \mathrm{~ms})$, the prime $(67 \mathrm{~ms})$, the target (initially $67 \mathrm{~ms}$ ), the mask ( $200 \mathrm{~ms})$, a blank interval $(400 \mathrm{~ms})$, and then the forced-choice display followed by feedback. Before each trial began, all stimuli were written into off-screen bitmaps; during the trial they were copied to the screen within $20 \mathrm{~ms}$ using the same transfer routine in all conditions. The initial mask served as a warning signal, and subjects were instructed to fixate on the cross in its center. Then the prime and target appeared in sequence, centered on a randomly determined corner of the imaginary square, followed by the mask. When the forced-choice screen (with three alternatives only) was presented, subjects used a mouse to click on the name of the object they thought was presented. The forced-choice display was then erased and feedback was given: a 250-ms pleasant sound for correct responses and a 750-ms less pleasant sound for incorrect responses. Thirty-three milliseconds after the feedback, the next trial began with the presentation of the mask and fixation point.

Each block consisted of 30 trials, one for each combination of object and priming condition. Trial types were randomly ordered within a block. There were 17 blocks, the first of which was treated as practice. After each block, the stimulus durations were adjusted depending on the level of performance during that block, in $17-\mathrm{ms}$ increments. The target durations were increased if performance fell below $50 \%$ and decreased if performance rose above $70 \%$, within fixed limits of 33 and $100 \mathrm{~ms}$.

Subjects were tested individually in sessions lasting approximately $50 \mathrm{~min}$. Viewing distance was maintained at approximately $70 \mathrm{~cm}$ by constraining chair position and instructing the subject to sit upright. Before the first block, instructions were read to the subject and each object was pointed out to the subject using the full (six alternatives) forced-choice display. Then the subject completed six instructional trials during which the target duration was increased to $500 \mathrm{~ms}$. These trials helped subjects to understand the stimulus sequence. Then the target duration was adjusted to its initial setting $(67 \mathrm{~ms})$ and the practice block began. Nothing was said to the subjects about the primes.

Subjects. Twenty-three subjects participated. They were from introductory psychology courses at the University of South Florida and received extra course credit for their participation. 
(a)
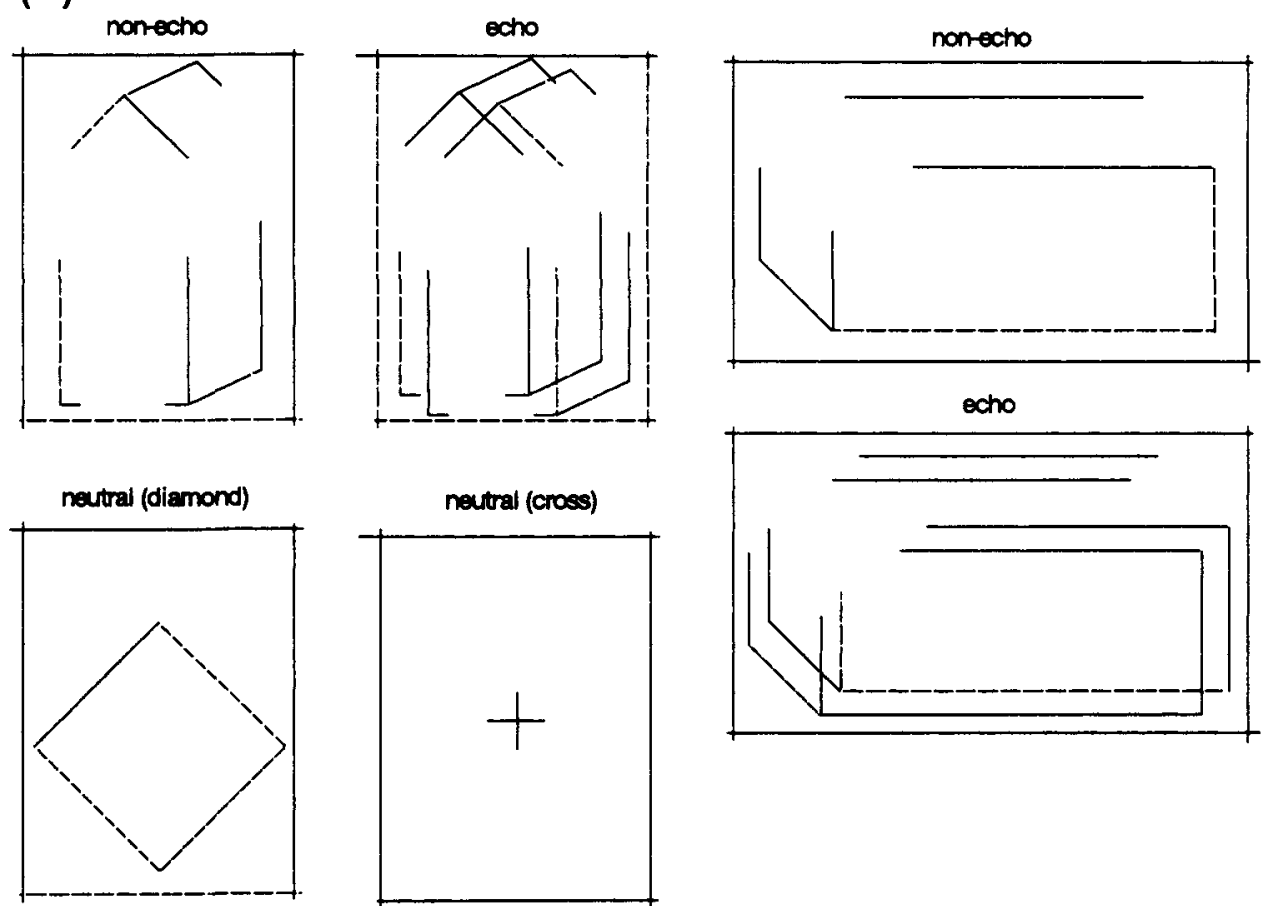

(b)
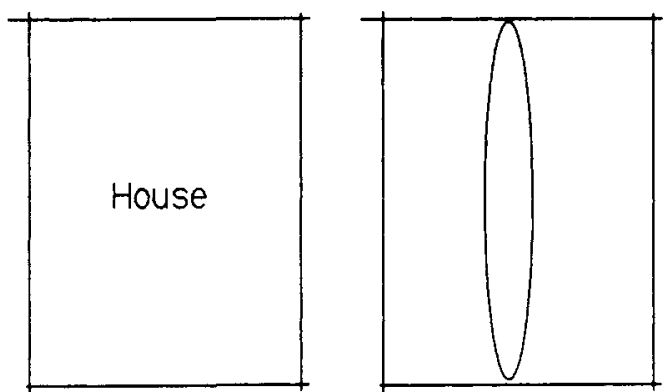

Figure 3. Panel a shows the primes used in Experiment la. (As noted in the Method section, there were two versions of each neutral prime, only one of which is shown.) Panel b shows examples of the new primes used in Experiment $1 b$.

\section{Results}

At the end of the experiment, the average target duration was $43.5 \mathrm{~ms}$. Analyses were conducted on subjects' mean percentages correct in each prime condition. The primary concern was the amount of facilitation and inhibition for consistent and inconsistent primes relative to neutral primes. Performance in the two neutral conditions was very similar, so they were combined in the analyses: For the diamond, $M=63.0$, and for the cross, $M=62.3, F(1,22)<1$, $M S_{\mathrm{e}}=83.7$.

Facilitation and inhibition effects (primed - neutral) are shown in Figure 4. The facilitation effects were reliable for both the consistent echo and nonecho primes: For the nonecho primes, $F(1,22)=7.22, M S_{\mathrm{e}}=141.0, p<.05$; for the echoes, $F(1,22)=14.90, M S_{\mathrm{e}}=178.0, p<.01$. These facilitation effects support the contingency hypothesis, but were not predicted by the general feature model. The facilitation effect was slightly (2.0\%) larger for the echo primes than for the nonecho primes, but these two prime conditions were not reliably different, $F(1,22)=2.03, M S_{\mathrm{e}}=141.4$, $p>.10$. The fact that the echo primes were at least as effective as the nonecho primes supports the claim that precise spatial information about the target is not necessary for facilitation. This result is consistent with the global-to-local model.

The inconsistent, nonecho primes had an inhibitory effect, $F(1,22)=15.91, M S_{\mathrm{e}}=160.8, p<.001$. This effect is addressed in the Discussion section. 


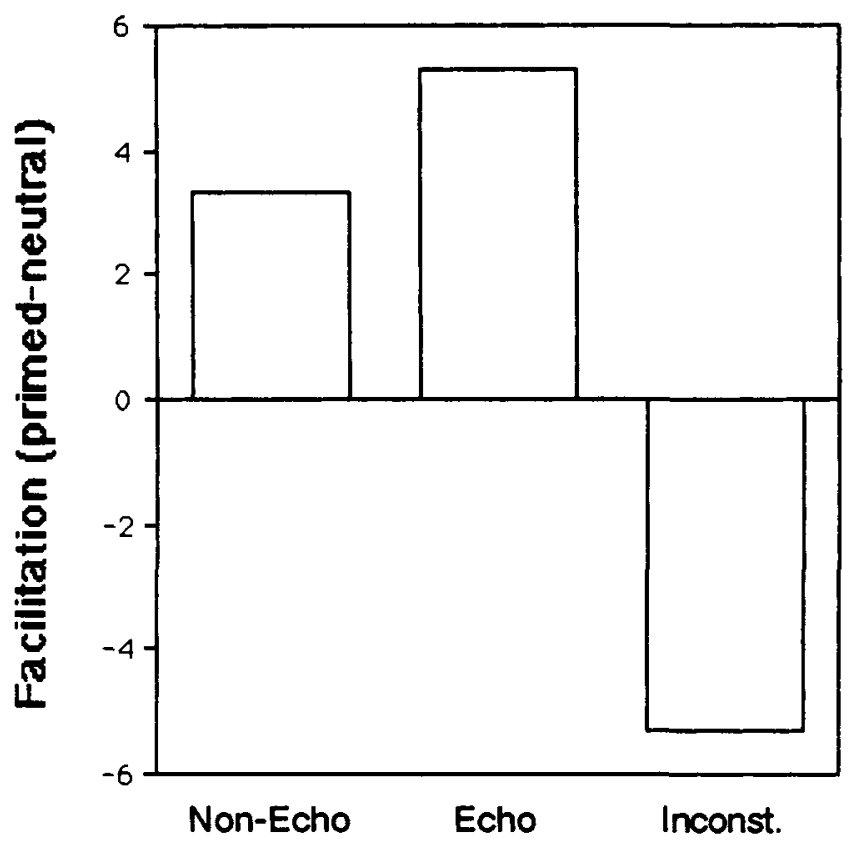

\section{Prime Type}

Figure 4. The amount of facilitation or inhibition in each prime condition of Experiment 1a. (Inconst. = inconsistent.)

\section{Experiment 1b}

In the contingency hypothesis, the effects of common feature primes are visual in nature. However, the primes could also provide semantic information about the class of the target, and consistent semantic information could have caused the facilitation effects. To test this possibility, Experiment $1 \mathrm{~b}$ was conducted with two new types of primes that gave information about the class of the target but less visual information. One type of prime was a word prime ("house" or "vehicle"). The other type of prime was a gross-shape prime that gave the target's dominant orientation, which was confounded with shape class. If the primes facilitated identification by providing information about the target's class, then at least one of the two new prime types would produce facilitation. On the other hand, if the main effect of the primes was to provide information about the visual structure of the objects (as posited in the contingency hypothesis and globalto-local model), then neither of these new primes would produce strong priming effects relative to a neutral prime. A common-feature prime was also used in the experiment, to establish that priming effects could be obtained in the experiment. All primes were consistent with the target, to maximize their validity.

\section{Method}

Examples of the new prime types are shown in Figure 3b. The word primes ("House" and "Vehicle") were approximately $.94^{\circ}$ and $1.31^{\circ}$ in length, respectively, and the large letters were $.24^{\circ}$ in height. The orientation primes were vertical and horizontal ovals approximately $3.4^{\circ}$ and $4.9^{\circ}$ in length, respectively. The neutral prime was the cross from Experiment 1a. The consistent prime was a near-echo prime, illustrated and discussed in relation to Experiment 3 . It provided fairly precise information about the targets' common features. In each block, each target object was preceded once by each type of prime, for a total of 24 trials. There were 19 blocks, the first of which was practice. Otherwise, the apparatus and procedure were the same as in Experiment 1a. A new sample of 18 subjects from the same population participated.

\section{Results}

The average target duration at the end of the experiment was $38.0 \mathrm{~ms}$. Performance in the neutral prime condition was $61.6 \%$. Facilitation was expected from the common feature primes, and the $5.1 \%$ facilitation effect was indeed reliable, $F(1,17)=9.88, M S_{\mathrm{e}}=241.3, p<.01$. For the word primes, there was no facilitation $(-1.0 \%$ effect). For the orientation prime, the $2.5 \%$ facilitation effect was not reliable, $F(1$, $17)=2.24, M S_{\mathrm{e}}=154.5, p>.10$. This small (and unreliable) facilitation effect may be caused by the partial visual information provided by the primes' orientations. Overall, the results indicate that the facilitation from consistent primes in Experiment 1a cannot be attributed to semantic information provided by those primes.

\section{Discussion of Experiments 1a and $1 \mathrm{~b}$}

In Experiment 1a, primes containing common global shape features facilitated identification. These effects are consistent with the idea of time course contingencies but were not predicted by the feature model. The common-feature facilitation effect was replicated in Experiment 1b, but primes that provided mainly semantic information about the class of the target did not cause facilitation, providing evidence against explanations of the facilitation effects in terms of semantic processing.

In Experiment 1a, the echo primes were found to be as effective as the nonecho primes, suggesting that precise spatial information about the target is not necessary for the facilitation effect. This result is consistent with the global-tolocal model. However, conclusions regarding the grain size of early analyses must be viewed as tentative for at least two reasons. First, the echo primes did contain precise information (i.e., sharp lines); the echo manipulation only moved the information away from target locations. The conclusion that precise target information is not necessary for facilitation would be stronger had facilitation been obtained with primes that had no sharp discontinuities. (This possibility was examined in Experiment 2.) Second, the echo primes contained twice as much information (i.e., twice as many lines) as the nonecho primes. (In Experiment 3, amount of information was equated across level of prime precision.)

A third finding in Experiment la was that inconsistent primes inhibited performance. The effect can be explained in at least two ways. First, inconsistent primes may activate representations or interpretations inappropriate for the tar- 
gets, thereby reducing the efficiency of later processing. Second, inconsistent primes may mask the targets. It is also possible that these two explanations are two ways of describing the same phenomenon. The present inhibitory effect contrasts with the previous experiments with two-dimensional patterns, in which no inhibitory effects were obtained (Sanocki, 1991a). There are several reasons why the difference may have occurred. First, it may be that contingencies or masking effects are stronger with complex objects than with simple patterns because more resources are required with objects. For example, an object prime may draw more resources away from interpretations consistent with the target than a pattern prime. Second, the stimuli were presented parafoveally and premasked in the present experiment but not in the previous experiments. Third, the difference could be due to the different control or neutral conditions used.

\section{Experiment 2}

The main purpose of Experiment 2 was to attempt to replicate the facilitative effects of consistent but imprecise primes with fuzzy primes that contained no sharp discontinuities. If such primes were effective, it would provide further support for the claim that precise spatial information about the target is not necessary for the facilitation. Inconsistent fuzzy primes were also used in Experiment 2.

\section{Method}

Fuzzy primes were generated by taking the echo primes from Experiment 1a and filling in the area between the echoes. A lighter pattern was used on the edges and a darker pattern in the center; a piece of contour is shown in Figure 5. The patterns were Macintosh Patterns 10 and 9, respectively (Inside Macintosh, 1985). Because the patterns themselves were fuzzy, this created a fairly gradual increase in darkness from the edge to the middle of the echo. There were three prime conditions in this experiment: consistent and inconsistent fuzzy primes and a neutral prime (the cross from Experiment 1a). In each block, each target object was preceded twice by each type of prime, for a total of 36 trials. There were 15 blocks, the first of which was practice. A new sample of 11 subjects from the same population participated.

\section{Results}

The average target duration at the end of the experiment was $39.4 \mathrm{~ms}$. Facilitation and inhibition effects are shown in Figure 6 (the neutral mean was $65.2 \mathrm{~ms}$ ). The facilitation effect for consistent primes predicted by the contingency hypothesis was obtained again, $F(1,10)=9.75, M S_{\mathrm{e}}=$ 203.4, $p<.05$, indicating that precise spatial information was not necessary for facilitation to occur.

Inconsistent primes caused inhibition, $F(1,10)=8.85$, $M S_{\mathrm{e}}=211.0, p<.05$. This replicates the finding in Experiment $1 \mathrm{a}$ and can be explained within the contingency hypothesis or in terms of masking.

\section{Experiment 3}

Experiments 1 and 2 established that precise spatial information about the target is not necessary for a facilitation

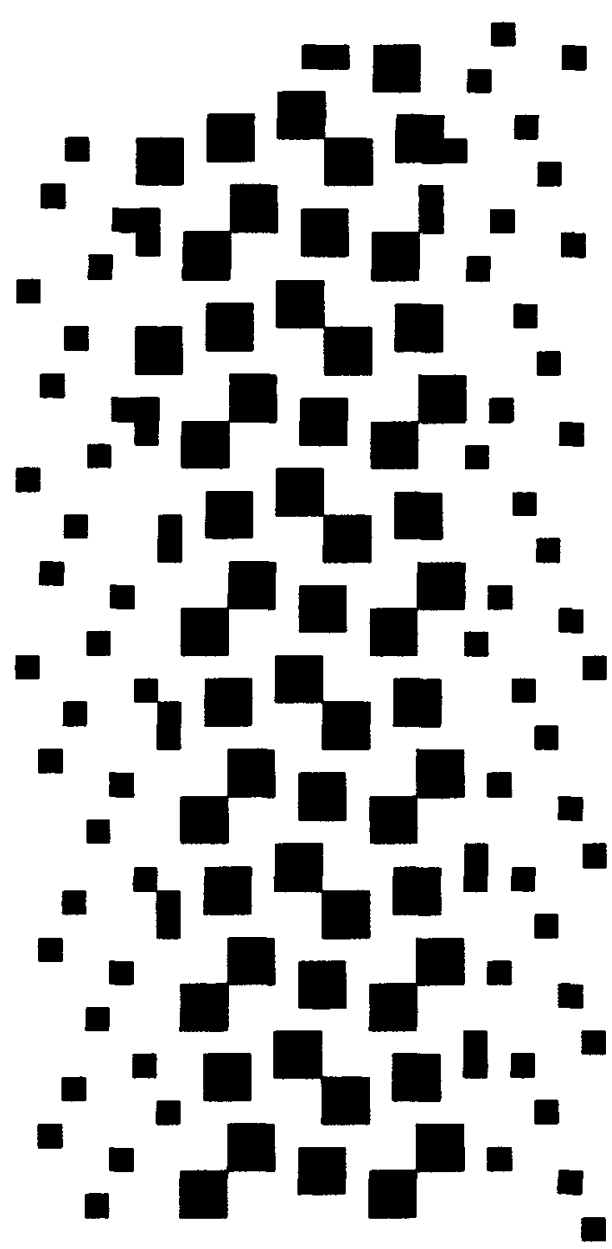

Figure 5. A piece of the fuzzy line used in Experiment 2.

effect. However, the results did not rule out the possibility that precise information contributes to the facilitation effect. The goal of Experiment 3 was to determine which types of information contribute to the effect, by using a continuum of primes varying in precision. Echo primes were used because they permit the amount of information (number of lines) to be equated across degree of precision. There were three levels of echo: near ( 2 min separation), medium (14 $\mathrm{min}$ ), and far $(28 \mathrm{~min})$. Only consistent primes were used. Near-echo primes contained most of the fine-grained information of the target, as well as medium- and large-grained information. As echo size increased, target-relevant information at grain sizes smaller than the echo size was filtered out. The grain size of the early analyses in Experiment 3 was expected to be revealed by the shape of the function relating amount of facilitation to degree of echo.

Five possible functions are shown in Figure 7. Function $A$ is flat, indicating that no target-relevant information was filtered out by the echos. This would imply that only large grain-size analyzers (larger than the largest echo size) were involved in early vision in the present situation. In Function $B$, facilitation falls off for far primes only, but not to zero. This would indicate that large echos filtered out some but not 


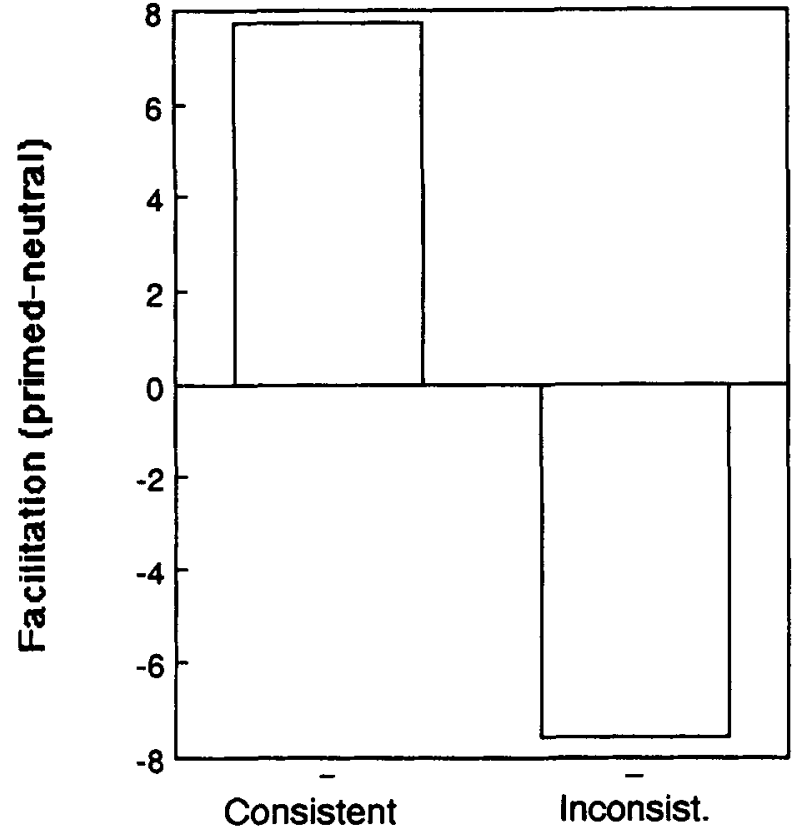

Prime Type

Figure 6. The amount of facilitation or inhibition in each prime condition of Experiment 2. (Inconsist. = inconsistent.)

all target-relevant information, implying that in Experiment 3 early vision involved both medium analyzers (whose information was blocked by the large echo) and large analyzers. The shape of Function $\mathrm{C}$ is similar to that of Function $\mathrm{B}$, except there is no facilitation with far primes. This would indicate that large echos filtered out all of the relevant information, implying that only medium analyzers were involved. The last two functions were unlikely, given the results of Experiments 1 and 2. In Function D, facilitation falls off gradually to zero as precision decreases, implying that small and medium analyzers were involved. In Function E, facilitation falls to zero with medium primes, implying that only fine-grained analyzers were involved.

The attention-attracting effects of neutral primes were explored further in Experiment 3. Specifically, the amount of information (number of lines) in or area of a prime may be relevant factors. In order to examine this issue further, a new large prime was used in addition to the cross prime. The large prime consisted of approximately as many lines as the content primes, and the lines formed the edges of a rectangle whose dimensions were intermediate between that of the houses and vehicles. If amount of information or stimulus area effects attentional capture, then performance should be higher with the large prime than with the cross prime.

\section{Method}

Examples of the primes are shown in Figure 8. There was a total of five prime types: near, medium, and far echos (all of which were consistent) and cross and large neutral primes. There was one version of the large neutral prime; it was approximately $4.2^{\circ}$ in width and $2.9^{\circ}$ in height and was positioned in a new large rectangle. The large prime was centered with respect to all six target objects, and its contours were positioned so they overlapped minimally with the major contours of the targets. In each block, each of the six target objects was preceded once by each type of prime, for a total of 30 trials. There were 17 blocks, the first of which was practice. A new sample of 22 subjects participated.

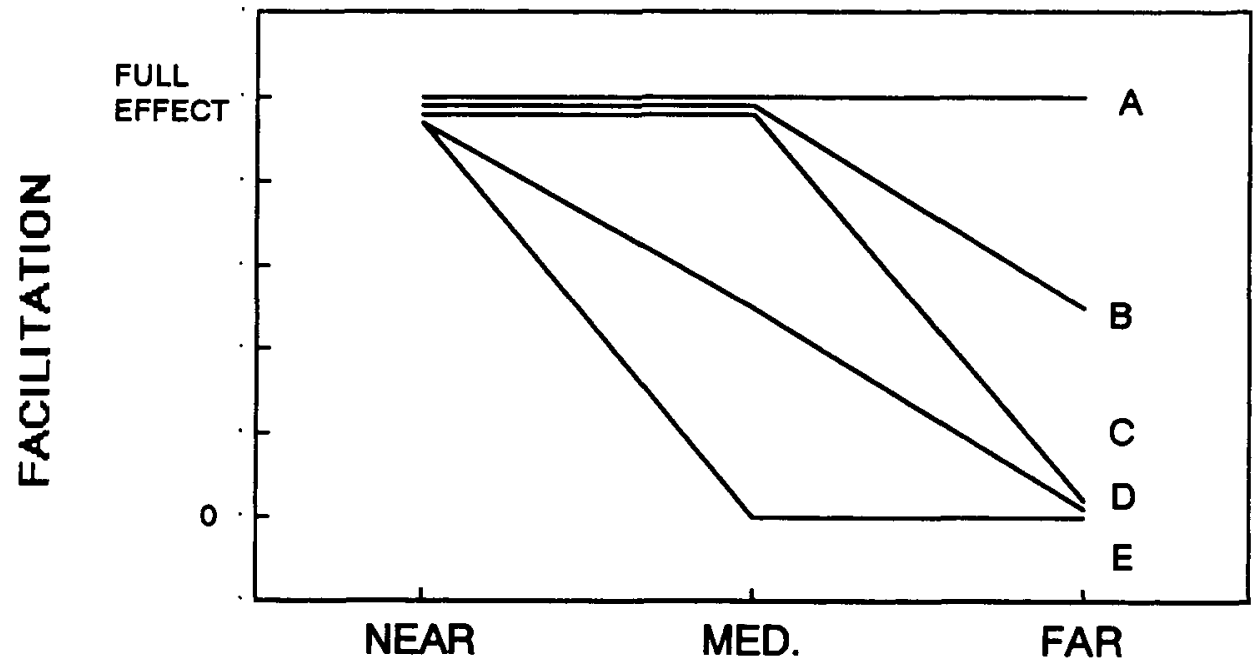

Echo Size

Figure 7. Possible functions relating the amount of facilitation to the degree of echo in Experiment 3. $($ MED. $=$ medium.) 

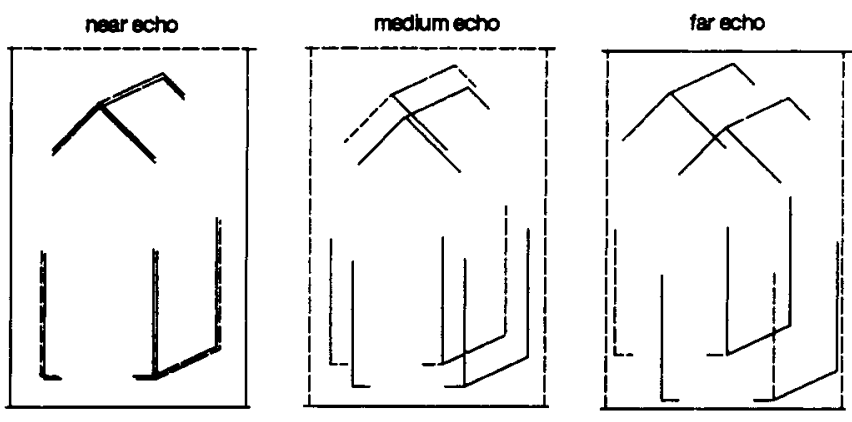

lange noutral

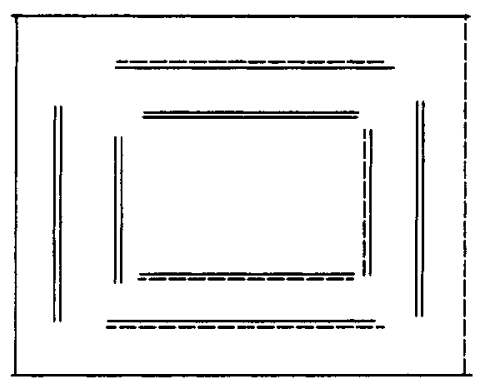

Figure 8. Examples of primes used in Experiment 3.

\section{Results}

At the end of the experiment, the mean target duration was $37.1 \mathrm{~ms}$. Performance following the two neutral primes was similar and so the data from these two conditions were combined in the analyses: For the large prime, $M=62.5$, and for the cross, $M=63.1, F(1,21)<1, M S_{\mathrm{e}}=126.4$.

Facilitation effects for the three types of echo primes are shown in Figure 9. As can be seen, there were substantial

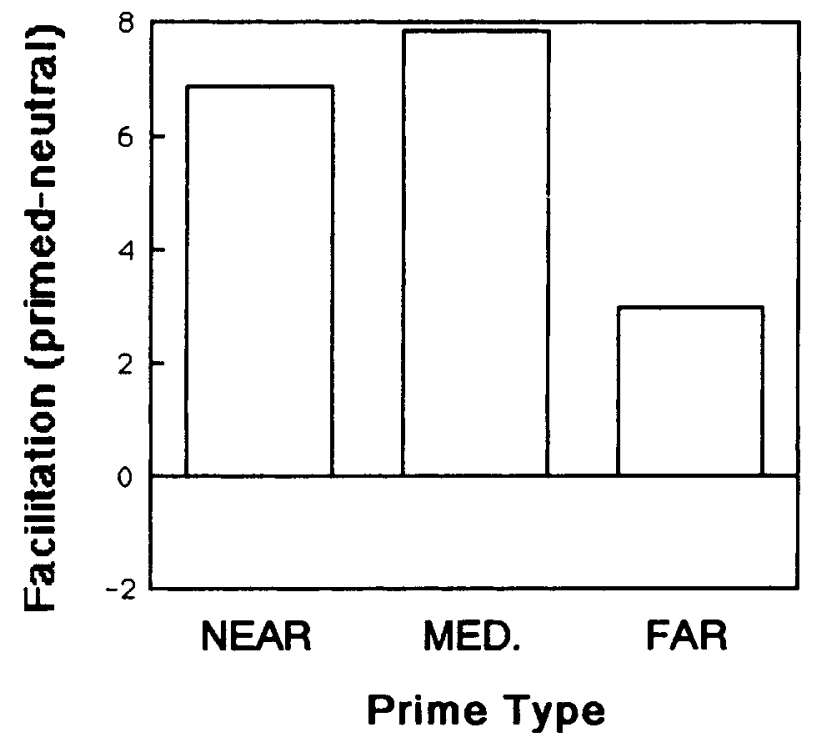

Figure 9. The amount of facilitation in each prime condition of Experiment 3. (MED. = medium.) facilitation effects for near- and medium-echo primes, but the effect began to fall off with far echos. The facilitation effects for the near and medium echos were highly reliable: For the near echo, $F(1,21)=33.18, M S_{\mathrm{e}}=125.9, p<.001$; for the medium echo, $F(1,21)=50.71, M S_{\mathrm{e}}=106.7, p<.001$. For far primes, the effect was reliable only in a one-tailed test: $F(1,21)=3.45, M S_{\mathrm{e}}=223.3, .10>p>.05$. The difference between the near and medium primes was small and unreliable, $F(1,21)<1, M S_{\mathrm{e}}=121.0$, but these two primes differed reliably from the far primes, $F(1,21)=8.79$, $M S_{\mathrm{e}}=194.2, p<.01$. Thus, facilitation fell off as echo size increased beyond medium. However, it did not fall off to zero for far primes (the effect was closer to being reliable than to being zero). These results are most consistent with Function $B$ in Figure 5, suggesting that the early analyses in Experiment 3 involved medium and large grain-size analyzers.

\section{Discussion}

The main finding of Experiment 3 was that facilitation was constant across near and medium primes but began to fall off (but not to zero) with far primes. Together with the results of Experiments 1 and 2, this suggests that early processing under the conditions of these experiments involved medium analyzers (corresponding to a grain size of at least $14 \mathrm{~min}$ ) and large analyzers (corresponding to a grain size of at least $28 \mathrm{~min}$ ). This conclusion is consistent with the global-tolocal model.

If the analyzers that extract early information correspond to spatial frequency channels, then it may be possible to use echo primes to infer which channels are involved in early vision within a given experimental situation. However, such inferences are complex. The response of a spatial frequency channel varies sinusoidally, with maximal sensitivity to information lying near the center of the channel (where a sinusoidal function peaks). Therefore, if a given channel responds as strongly to medium echos as to near echos, the positive portion of its response function must be wider than the medium echo, in order for the contours to fall near its center. Thus, the wavelength is likely to be several times the echo size. Furthermore, the relative sensitivity of spatial frequency channels may vary with a number of factors, including retinal eccentricity, luminance, and stimulus duration (for evidence with near-threshold patterns, see Graham, 1989).

Although the present results follow directly from the global-to-local model, other possible influences should be examined in future research. For example, the effectiveness of a prime might be determined by its ability to convey depth. However, some evidence against the importance of depth was provided by Experiment 4, in which global primes were quite effective even though they contained less depth information than the present primes. The primes in Experiment 4 contained mainly exterior contours, conveying the target's silhouette, whereas the primes in Experiment 3 contained some interior contours and vertices that conveyed more shape and depth information. Also, in more recent experiments, equal facilitation effects were obtained with primes differing in amount of depth information. A second factor to consider is lateral masking: Primes that do not match target 
contours precisely may mask targets more than matching primes. However, it seems such interference was minimal in the present consistent-prime conditions, because the nonecho primes in Experiment 1, which matched precisely, were not more effective than the medium-echo primes in Experiments 1 and 3 or the near-echo primes in Experiment 3. A third factor that may be important is the parsability of the primes. It may be easier to group corresponding contours when they are closer together (e.g., near echoes) than when they are farther apart (e.g., far echoes). However, the relation between parsability and separation would not seem to be linear, because near-echo primes were not superior to medium-echo primes.

\section{Experiment 4}

Experiments 1-3 supported the contingency hypothesis by showing that early common shape features facilitate object identification and supported the global-to-local model by suggesting that early analyses use medium and large grainsize information. In the global-to-local model, the facilitation effects occur because early information activates a representation that is useful during later processing.

In Experiment 4, a further prediction of the contingency hypothesis and the global-to-local model was tested: that the relative contribution of different types of information to the eventual percept would change over time. Early in processing, global information should contribute more than local information because it can be used to begin constructing a global representation. Once the representation is constructed, local information should contribute more because it can be readily interpreted. The local information would serve to distinguish one alternative from others.

To test this prediction, the order of primes and targets was varied in Experiment 4: On a given trial, the prime either preceded the target (prime-TARGET trials, as previously), or the prime followed the target (TARGET-prime trials). (TARGET is capitalized to indicate that targets contained more complete information than primes.)

The second major change was that both global and local primes were used. Global was operationalized as exterior contours (including exterior vertices), whereas local was operationalized as internal (and generally smaller) contours, for reasons given shortly. Also, given the results of Experiments $1-3$, the global primes were medium echo. The local primes were nonecho primes. Because the general feature model was no longer being tested, the global and local primes were not limited to common features. To maximize the power of the priming manipulation, one global and one local prime were generated from each object, and both primes included distinctive features. The global primes had some distinctive exterior features, and most of the local contours of the local primes were distinctive.

Although there are alternative ways to operationalize global (vs. local), the present emphasis on externality (vs. internality) was reasonable for initial tests of the present prediction, because a number of considerations imply that exterior features should be important early in processing. First, exterior contours of naturally occurring objects are likely to be highly discriminable because they are often accompanied by changes in texture and depth (between figure and ground), and because when an object moves (against ground) the edges are accompanied by differences in motion. Second, Waltz (1975) examined the relative ambiguity of interior and exterior edges in a blocks world environment and concluded that exterior edges were initially less ambiguous. Therefore, exterior edges would provide a more reliable starting point in processing. Third, if the exterior contour is known, then the spatial extent of processing is known and resources can be devoted to the region within that contour. Fourth, the exterior outline is generally larger than interior features; larger objects may be more ecologically significant, and larger components often are more central to the overall structure of an object. Fifth, object shape can be a highly diagnostic cue to object identity (with rigid and at least some nonrigid objects). For these same reasons, interior contours are generally less important early in processing. Consistent with this argument, several investigators have obtained perceptual or attentional advantages for exterior lines over interior lines (e.g., Earhard \& Walker, 1985; McClelland \& Miller, 1979). Qualitatively different priming effects for global and local primes would provide further validation of the distinction between global and local features.

\section{Method}

The global and local primes are shown in Figures 10 and 11. As noted, there were one global and one local prime for each object. An attempt was made to equate the global and local primes for overall effectiveness; this was done by using approximately similar total line lengths in the initial versions of the primes and then making small adjustments in the primes based on overall performance levels for each prime during pilot work. The neutral prime was the cross. In the prime-TARGET conditions, the target was preceded by a global, local, or neutral prime (global and local primes were always consistent). The same three types of primes occurred in the TARGET-prime conditions, but the order of the prime and target was reversed.

In each block, each target object was used once in each of the six order-prime conditions, for a total of 36 trials. There were 15 blocks of trials, the first of which was treated as practice. The initial durations were $83 \mathrm{~ms}$ for the primes and $67 \mathrm{~ms}$ for the targets. As previously, durations were adjusted on the basis of performance in each block. However, both the target and prime durations were adjusted, in 17-ms increments, within limits of 33 and $100 \mathrm{~ms}$. The criteria for adjusting durations in the present experiment were widened a total of $10 \%$ to $45 \%$ and $75 \%$, because the total size of stimulus adjustments was $33 \mathrm{~ms}$ in the present experiment. A new sample of 17 subjects from the same population participated.

\section{Results}

The mean target duration was $40.2 \mathrm{~ms}$ at the end of the experiment. Of primary concern was the predicted interaction of the two content primes (global vs. local) and order. Consistent with the contingency hypothesis and the globalto-local model, there was a rather striking interaction: Global primes were $5.9 \%$ more effective than local primes when the primes were presented first, but $9.2 \%$ less effective when the primes were presented second, $F(1,16)=22.23, M S_{\mathrm{e}}=$ 
264.6, $p<.001$. This result suggests that the relative contribution of global and local information changed over the time course of object identification.

More detailed analyses required separating the data by stimulus order, because the second stimulus in the sequences was more effective. The advantage for the second stimulus was seen most clearly in the neutral prime conditions: Performance was higher in the neutral-TARGET condition $(69.0 \%)$ than in the TARGET-neutral condition (47.0\%), $F(1,16)=93.96, M S_{\mathrm{e}}=262.5, p<.001$. At least two factors may have contributed to the advantage for the second stimulus. First, the premask is likely to have interfered with the first stimulus. Second, if attentional resources were shifted to the target location over time, then more resources would have been available at the onset of the second stimulus than at the onset of the first stimulus, because the shifting process would have begun only after the onset of the first stimulus.

Figure 12 contains the amounts of facilitation for global and local primes relative to neutral primes in the appropriate stimulus order. When the prime was the first stimulus (left panel), global primes had a highly reliable facilitation effect, $F(1,16)=18.63, M S_{e}=244.7, p<.001$, whereas the effect for local primes was reliable only in a one-tailed test, $F(1$, 16) $=3.43, M S_{\mathrm{e}}=182.5, .10>p>.05$. Performance was reliably higher for global primes than for local primes, $F(1$, 16) $=13.62, M S_{\mathrm{e}}=132.7, p<.01$. When the prime was the second stimulus (right panel), both global and local
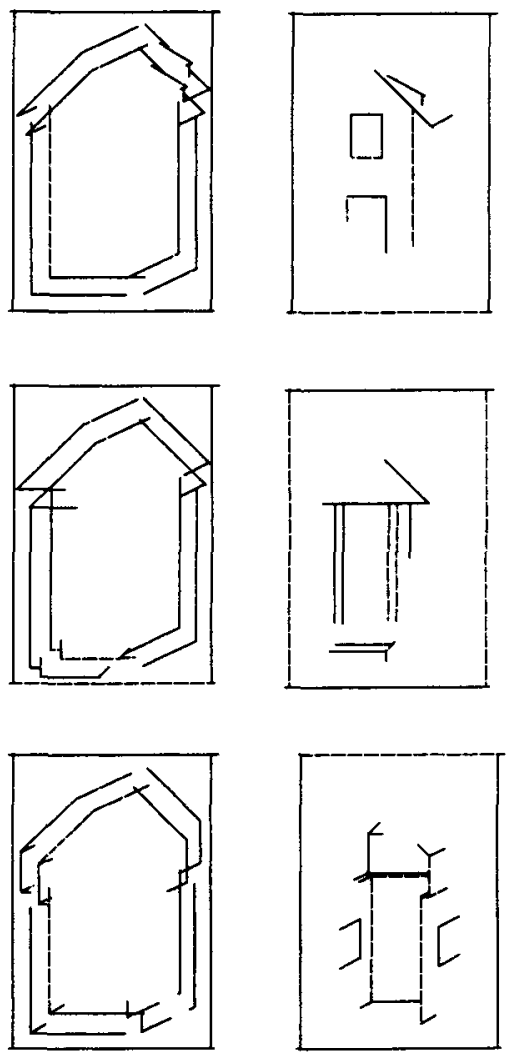

Figure 10. Primes used for house targets in Experiment 4. (Left column, global primes; right column, local primes.)
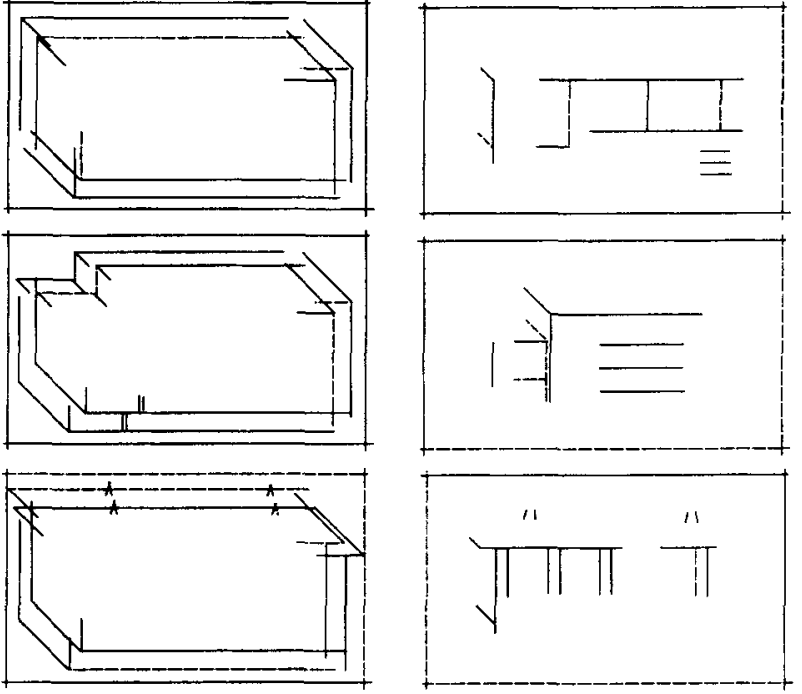

Figure 11. Primes used for vehicle targets in Experiment 4. (Left column, global primes; right column, local primes.)

primes had highly reliable facilitation effects: For global primes, $F(1,16)=21.56, M S_{\mathrm{e}}=224.3, p<.001$; for local primes, $F(1,16)=77.51, M S_{\mathrm{e}}=236.8, p<.001$. However, more important is the fact that performance was higher with local primes than with global primes, $F(1,16)=8.12$, $M S_{\mathrm{e}}=536.0, p<.05$.

\section{Discussion}

The results of Experiment 4 provide particularly strong support for the idea of time course contingencies and for the global-to-local model: The relative effectiveness of global and local information reversed over processing time. Overall processing was most efficient when it began with global information and when it ended with local information. These results suggest that there is a global-to-local trajectory in the development of a percept.

Although the effect of early local primes was only marginally significant in Experiment 4, the present claim is not that local information is ignored in early processing. In the present situation, local details are likely to have a strong impact on performance because they can distinguish between alternatives. The priming effect would be likely to become more reliable if the amount of information in the local primes was increased. The present claim is that the usefulness of local information changes over time, with local information becoming more useful later in processing, after a global representation has been established.

In Experiment 4, there were differences in the effectiveness of the first and second stimuli in the sequence. However, the crossover nature of the crucial Prime $\times$ Order interaction seems to rule out explanations of the interaction in terms of differences in level of performance. In more recent experiments discussed later, performance was equated between the two orders and similar interactions between globality and order were obtained. 

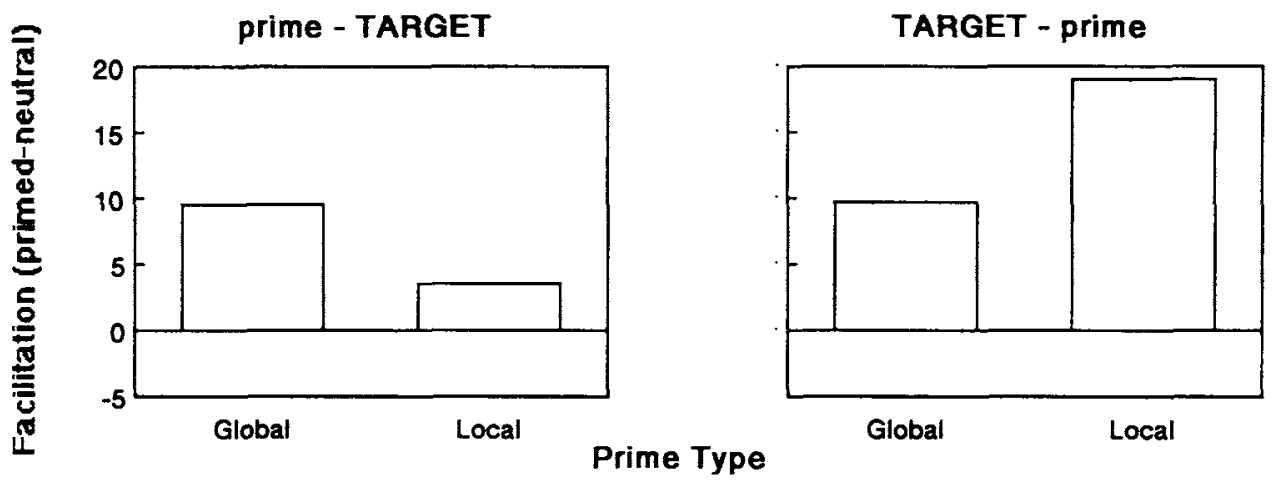

Figure 12. The amount of facilitation in each prime and order condition of Experiment 4.

The qualitatively different effects of global and local primes provide some validation of the distinction between global and local features. However, in the attempt to maximize the power of the priming manipulation, several factors were confounded with globality (vs. locality). Relative to local primes, the global primes were less precise, larger in grain size and somewhat larger in scale, and more exterior. These properties should be distinguished in further research. Also, although the effect of global primes appeared to be more constant than the effect of local primes across time (see Figure 12), these differences should be interpreted with caution. Global primes contained some detailed outline features, which may have functioned as local features, and thus may have been useful during later processing. In addition, although the present experiments measured early processing, it is not clear how much of late processing was measured, because the mask interfered with processing before it was complete. Global primes may become less effective during processing intervals beyond those examined in the present experiments. Finally, as noted, overall accuracy levels differed between the two conditions in Experiment 4. In some more recent experiments, the duration of the first stimulus was increased to compensate for its lower perceptibility, and global priming effects were reduced in the TARGET-prime order relative to the other order. For example, in the experiment most similar to Experiment 4, 17 ms were added to the duration of the first stimulus on every trial. Performance was $59 \%$ in the neutral-TARGET condition and $61 \%$ in the TARGET-neutral condition, indicating that the first stimulus was at least as perceptible as the second one. Global priming effects were reduced over time, from $14 \%$ to $8 \%$ in the prime-TARGET and TARGET-prime conditions, respectively. Local priming effects increased over time, from $10 \%$ to $14 \%$ in the respective conditions. These results (as well as the results of several other experiments) replicate the general pattern of effects obtained here.

\section{General Discussion}

\section{The Contingency Hypothesis}

In the contingency hypothesis, early stimulus information is used to increase the efficiency of processing later consis- tent information. The nature of processing changes over time from being based more on general information early in processing to being based more on detailed, item-specific information later in processing. This helps to reduce the computational burden of processing detailed information about objects. The contingency hypothesis has been expressed in a general manner because there are a number of possible mechanisms by which time course contingencies might operate, and thus the hypothesis represents a class of models. Some contingency mechanisms not considered in the present experiments are discussed in the following sections.

A major prediction of the contingency hypothesis confirmed in Experiments 1-3 is that common-feature primes facilitate identification because the early information increases the efficiency of later processing. This occurred even though the primes per se contained no information that would have aided in the forced-choice discriminations. Experiment $1 \mathrm{~b}$ indicated that the priming effect cannot be attributed to semantic information about the target's class provided by the prime. The facilitation effects do not seem attributable to errors in the baseline estimates, because there was little variation in the baseline levels produced by the three different neutral primes used in Experiments 1 and 3. A second major prediction of the contingency hypothesis is that the relative contribution of different types of information to the eventual percept change over time; this was confirmed in Experiment 4.

More specific predictions were confirmed for one instantiation of the contingency hypothesis, the global-to-local model. In this model, early global information is used to build a coarse representation that is useful in later, more local processing. Consistent with this model, the facilitation effects in Experiments 1-3 can be attributed to the processing of early but imprecise global information. A second supportive finding was that when the relative contribution of different types of information changed over time (Experiment 4), the change was from global to local information. This suggests that there is a global-to-local trajectory in the development of a percept.

The qualitatively different effects of different types of features partially validates a distinction between global and local features. Further research with the integration priming paradigm into qualitative differences could provide further 
information about the nature of perceptual features. Of particular interest is the implication of Experiment 4 that the features used in perception are to some extent context or time dependent.

The finding that the relative contribution of global and local information reverses over time is a new example of global-to-local processing that is not easily accounted for within alternative explanations of the global precedence effects discovered by Navon (1977) with hierarchical forms. The present finding cannot be explained in terms of differences in discriminability between global and local levels (e.g., Pomerantz, 1983), because the crucial effect was a reversal in effectiveness over time rather than an overall advantage for one of two distinct processing and response levels. The present finding is not easily attributed to differences in attentional or decisional priority for global and local levels (e.g., Miller, 1981), because there was only one decision level in the present task. ${ }^{3}$

The present conclusion that global information is important in early processing is consistent with the idea of global precedence supported in experiments with hierarchical forms (e.g., Navon, 1977). However, there are at least two different views on the relations between the psychological processes probed within these two paradigms. The two paradigms may tap similar processes. Alternatively, the paradigms may tap different processes. In particular, the present effects may result from a single object recognition process, whereas effects with hierarchical forms may result from attentional or decisional precedence for one recognition process (at the larger scale) over another independent process (at the smaller scale; see Boer \& Keuss, 1982; Miller, 1981; Sanocki, 1991b). A variant of the second view is that the two paradigms differ along a continuum. For example, the paradigms may differ mainly in the degree to which global and local levels are independent of each other.

The claim that the paradigms tap differing processes receives some support from the contrast in conclusions between the present studies and Navon's (1991) studies. As mentioned in the Introduction, Navon (1991) used hierarchical forms to examine the possibility of qualitative changes over time and found global precedence throughout the time course of processing. Global precedence may be more constant when there is greater independence between global and local levels, as in Navon's studies. (However, the paradigms differ in other ways, and these differences should also be considered.)

One other finding in Experiments 1 and 2 was that when the early information was inconsistent with the target object (inconsistent primes), inhibition occurred. This can be explained in terms of contingent processing based on the inconsistent prime or in terms of masking effects. However, similar inhibitory effects were not obtained with twodimensional patterns (Sanocki, 1991a). There are several possible reasons for the difference (see the Discussion section of Experiment 1). One explanation is that inhibitory effects are reduced with two-dimensional patterns because they require fewer processing resources than the present objects. In fact, such an explanation follows from a model discussed in the Constituency Model section.
The present findings follow directly from the contingency hypothesis and the global-to-local model, but it is important to consider alternative explanations and to consider the idea of time course contingencies more generally. This is done in the next section. As will be argued, none of the alternatives is entirely satisfactory, and the global-to-local model is also limited. Therefore, a generalization of the global-to-local model called the constituency model is briefly outlined. Then some models from somewhat different domains are discussed as possible further examples of time course contingencies.

\section{Some Alternative Explanations}

Interactive activation and programmable connections. One alternative to bottom-up feature models has been the interactive activation model (IAM; McClelland \& Rumelhart, 1981). In the IAM, letter units activate word units, which in turn provide top-down excitation for their constituent letter units. This is an example of a time course contingency in that early letter information determines which word units will become active, which in turn influences letter-level units. The idea of interactive activation might be extended to object identification by assuming that there are lower level units representing global and local features and higher level units representing objects. The common-feature facilitation effects in Experiments 1-3 might be explicable in terms of feedback from higher levels that begins soon after prime onset. However, it is not clear whether feedback would produce common-feature facilitation because it would support all similar-shape alternatives, not just the target. This demonstrates that top-down excitation may, in at least some cases, overpower stimulus information (cf. Massaro, 1989; McClelland, 1991).

The qualitatively different effects of global and local features across time (Experiment 4) appear to be clearly problematic for the IAM. Because features are treated uniformly in the model, there is no mechanism for differential effects. However, the IAM has recently been further developed in a number of ways, and some of the developments may be useful in the present case. In particular, McClelland (1985) developed a model in which the connections between lower and higher level units are activated dynamically (are "programmable") during perception, on the basis of early evidence at the higher level. The results of Experiment 4 could be explained if connections between object units and global feature units were active at the start of the trial, whereas connections between objects and more detailed local feature units became active only after the object units received stimulus-based activation. The idea of dynamic configuration is very much in the spirit of the contingency hypothesis. If dynamically allocated connections increase the sensitivity of the processing system to certain kinds of stimulus information, they could be quite useful in recognition. Although

\footnotetext{
${ }^{3}$ This is not to say that such an explanation cannot be constructed. It merely seems arbitrary and post hoc in the present context.
} 
the assumption of differences in connectivity for global and local features is arbitrary without further motivation, it could make sense within a model designed for object recognition. The IAM was not designed for object recognition, and its shortcomings in the present context should not be taken as evidence against the model.

Recognition by components. As noted in the introduction, Biederman (1987) elaborated on feature theories to create a powerful model of object identification. In his recognition by components (RBC) model, object identification begins with the extraction of features (nonaccidental properties), which then activate representations of parts, termed geons. Object representations are then accessed in a modelmatching process that uses geons and their relations. The specification of geons and their relations in RBC goes far beyond feature models of pattern recognition; however, the model is still bottom-up (except for edge extraction), and therefore there is no way for early results to modify later processing.

The RBC model would seem to have problems with the facilitation of common-feature primes in Experiments 1-3 because the model-matching process is based on overall similarity, as specified in Tversky's (1977) contrast model. In the contrast model, any facilitation of common features or geons would increase the similarity of the primed objects, which should reduce perceptibility. On the other hand, the changes over time in the effectiveness of global and local information obtained in Experiment 4 may be consistent with RBC. Biederman (1987) emphasized the importance of major geons, which in general are the largest geons. The global primes could be assumed to activate one or two major geons. Local primes, on the other hand, may activate minor geons or representations of surface features. If major geons are activated before minor geons or surface features, then the results might be explained in terms of the time course of activating global (large geon) and local (small geon and surface feature) representations.

More recently, Hummel and Biederman (1992) have developed an important extension of RBC named JIM (for "John and Irv's model"). JIM is a connectionist network that creates structural descriptions similar to those postulated in RBC, and it incorporates a number of important refinements. Of particular relevance here is a neural binding mechanism that causes units consistent with the same geon interpretation to fire synchronously, that is, to have the same phase relations between their activation spikes. Synchronism is used to bind together features of the same geon. Thus, synchronism becomes an additional but generally independent type of information used in processing. Neural binding is an interesting mechanism in its own right, and it could contribute to an explanation of common-feature facilitation. For example, binding might be facilitated by early information from common-feature primes and this may aid later processing enough to overcome the detrimental effects of activating common geons. However, the exact form such an explanation would take is not clear.

Three points can be made about JIM in the present context. First, neural binding creates a fuzzy boundary between strictly bottom-up processing schemes and time course con- tingencies. The binding mechanism uses information available early in processing to modify later processing. Specifically, information about the consistency of features with a particular geon determines which feature units will be grouped together to activate that geon. Thus, a piece of incoming information does not have unconstrained access to higher level representations because its effect depends on with what it is grouped. However, the flow of information is bottom-up, and JIM tends to use local information (but see the following).

Second, neural binding has a conjunction of properties that may be important in vision: It is closely tied to stimulus information and thus can maximize the utility of that information, while at the same time it exploits the emergent structural constraints of an evolving object percept.

A third point is that the implementation of JIM illustrates (some of) the computational complexity of object recognition. To create a structural description, the model uses five levels of representation and an apparently large number of units. Furthermore, only one of the three types of features (nonaccidental properties [NAPs]) used in JIM-the vertex type - is directly computed from local edge information. The second type of NAP (blob parameters, e.g., size and elongation) is computed from the results of a region-filling algorithm that uses global information. Such properties may be difficult to compute from local information. The third type of NAP (axis type, e.g., straight vs. curved) is not computed at all, but given as an input. Axis information is difficult to compute in general (note that axes are not literally in the object, but must be inferred from other information). The apparent difficulties of computing these types of information indicate that object recognition has some serious but nonobvious computational complexities and make the feasibility of a strictly local scheme questionable. To the degree that such complexities exist, time course contingencies may be warranted. For example, the difficulty of deriving axis information might be reduced if global shape information were used to constrain processes that infer axes.

The present comments should not be taken as criticism of the overall contribution of JIM. Model building would not be possible without assuming solutions to some problems, and JIM makes many important contributions. The point I wish to make is that object recognition involves serious complexities, for which contingencies are a possible solution.

Attentional explanations. Several distinct roles of attention may be considered. The first is a role that was controlled in the present experiments. The second and third are possible alternative explanations that do not involve contingencies.

First, shifts of attention through space can cause contingencies. That is, when early information (e.g., a cue) causes a shift of attention, subsequent processing in the attended-to location can be more efficient (e.g., Posner, 1980). This also fits the present definition of a contingency. For this reason, attention-shifting effects of primes were controlled in the present experiments by using several different neutral primes. However, the effects of attention and the way attention is shifted continue to be important areas of research and should not be overlooked as a possible source of contingencies. For example, the efficiency of processing a 
complex object could be increased by information (e.g., salient object structures) that guides the shifting of attention.

A second role of attention involves capacity limitations and provides a possible alternative explanation of some of the present results. Specifically, the effects of early commonfeature primes can be explained as follows. If the processing system is limited in capacity, then the head start in processing provided by an early prime would give the system additional time to extract features, and in particular features of the target that are not in the prime. Because those features tend to be distinctive, the increased likelihood of extracting them would increase accuracy relative to when there is no head start (neutral condition). However, capacity explanations have problems with the qualitative changes over time in Experiment 4, which indicated that features were differentially effective depending on when they were available; this is inconsistent with a simple capacity explanation, in which only the quantities of features and processing time are important.

A third role of attention involves the idea of abrupt onset. ${ }^{4}$ Recently, several investigators have found that stimuli with abrupt onsets are attended to and processed more quickly than stimuli with gradual onsets (e.g., Krumhansl, 1982; Yantis \& Jonides, 1984). In a typical experiment, an initial display contains one or more complete characters, obtained by combining the stimulus features from all targets. Next, features are gradually removed from the complete characters, to make identifiable targets (gradual onset), and then a new identifiable target appears instantaneously in a new location (abrupt onset). Subjects are quicker at identifying abruptonset targets than targets with gradual onsets, apparently because attention is attracted to the abrupt-onset stimulus (Yantis \& Jonides, 1984; cf. Miller, 1989). Such effects are potentially relevant in the present situation because the content of the first stimulus may have determined whether aspects of the second stimulus would appear abruptly (and thus attract attention). In particular, aspects of the second stimulus not in the first stimulus may appear abruptly. Consider the effects of early common-feature primes. When preceded by a common-feature prime, noncommon (distinctive) features of the target may have appeared abruptly and thus attracted attention. In contrast, with a neutral prime as the first stimulus, the entire target would have appeared abruptly, and both useful (distinctive) and nonuseful (common) features would have attracted attention. The more exclusive attention to distinctive features following common-feature primes could have produced facilitation.

However, there are questions about the applicability of abrupt-onset results in the present situation and about the ability of such an explanation to handle further aspects of the results. In most abrupt-onset experiments, the duration of the first stimulus was considerably longer (in general, $1 \mathrm{~s}$ in duration) than that of the first stimulus in the present experiments (in general, $70 \mathrm{~ms}$ or less). A long-duration initial stimulus is likely to be seen as a distinct entity, separate from the second stimulus. In contrast, when the duration of first stimulus is short (e.g., less than $80 \mathrm{~ms}$; Di Lollo, 1980), it is likely to be integrated into a single percept with the second stimulus. The general impression in the present experiments was of a single, integrated stimulus, although some changes in the stimulus over time could be observed. In one previous abrupt-onset study, the effects of variation in the duration of the first stimulus were examined. Krumhansl (1982) found that when the first stimulus had a long duration, it made an abrupt-onset second stimulus more perceptible than a nonabrupt stimulus. However, when the duration was less than $80 \mathrm{~ms}$, the perceptibility of the abrupt-onset stimulus tended to be reduced relative to that of a nonabrupt stimulus that was continuous with the first stimulus. Thus, with brief stimuli, the abrupt onset of a second stimulus may cause a perceptual disadvantage.

The abrupt-onset explanation also has problems with several aspects of Experiment 4 . In the explanation, the advantage for early global primes could be explained in the same way as the advantage for common-feature primes (i.e., distinctive local features of the target receive attention because of their abrupt onset). However, if features of the second stimulus not contained in the first stimulus are attended to, then it seems that when a local prime is the first stimulus (local prime-TARGET condition), it should interfere with processing, because global (less distinctive) aspects of the second stimulus would appear abruptly and receive increased attention, reducing attention to distinctive local aspects. Also, it is not clear how the strong priming effects in the two TARGET-prime conditions can be explained, given that neither the global or local prime appeared abruptly. ${ }^{5} \mathrm{~A}$ further possible problem for the abrupt-onset explanation is the facilitation effects found for medium-echo primes in Experiments 1,3 , and 4 . According to the abrupt-onset explanation, local differences between the first and second stimuli attract attention. However, there were local differences between an echo prime and the single contours of the target, and these should also have attracted attention. Thus, the entire target should have had an abrupt onset when it was preceded by an echo prime, and there should have been no advantage relative to the baseline condition. In sum, a simple abrupt-onset explanation does not appear capable of explaining the present results, and it is not clear what effect abrupt onsets would have in the present situation. Nevertheless, it may be important to consider this factor in future research.

Amount of time for processing global and local information. This type of explanation follows from the distinction between transient channels, which have fast response times and are more sensitive to lower spatial frequency information, and sustained channels, which have slow response times and are more sensitive to higher spatial frequency information (e.g., Breitmeyer, 1983). Transient channels could correspond to global information and sustained channels to local information. Although it would be difficult to evaluate such an explanation without a more detailed model, it does seem clear that the present results cannot be explained in terms of

\footnotetext{
${ }^{4}$ Lise Paquet suggested this as a possible explanation.

${ }^{5}$ One could argue that in Experiment 4, subjects attended mainly to the second stimulus, which was considerably more perceptible. Thus, content primes would have an advantage over the neutral prime when they were the second stimulus. However, in recent experiments, the same pattern of results held when the perceptibility of the first and second stimuli was equated.
} 
the amounts of time available for processing transient/global and sustained/local information. In Experiments 1-3, the priming manipulation increased the duration of global (transient) information but not local (sustained) information. However, in the sustained/transient dichotomy, more time would be necessary for processing local information. In Experiment 4 , the local prime-TARGET order allowed additional time for local processing, but performance was lower than that for the global prime-TARGET order.

Effects of semantic category. I have conceptualized the present manipulations mainly in terms of visual structure, rather than in terms of target category (which was confounded with visual structure in the present experiments). This emphasis on visual structure is supported by Experiment $1 \mathrm{~b}$, which provided evidence against the idea that early primes cause facilitation because they provide information about the class of the target. Thus, semantic information is not sufficient to cause facilitation. This conclusion is further supported by the fact that previous findings of commonfeature facilitation (Sanocki, 1991a) were obtained with letters or novel patterns, for which there were no category differences. Nevertheless, it is possible that within an experiment, subjects develop ad hoc categories, for example, "tall ones" or "short ones," for the stimuli. Such categorization may not be sufficient to cause facilitation, but it may be necessary for facilitation. That is, primes may cause facilitation only when they are relevant to items that form an ad hoc category. Thus, categorization should be considered in future research.

Global-to-local processing. The global-to-local processing model was successful in predicting the results of the present experiments. Briefly, the idea is that early information results in an imprecise global representation that is useful during the later processing of local information. However, the model is limited in several ways. First, it is not very specific. There are a number of possible reasons for the importance of global information early in processing (see introduction to Experiment 4; also Earhard \& Walker, 1985; Navon, 1991), but there is little evidence that points directly to specific mechanisms. Perhaps because of this, the definition of global (as well as local) information remains open. Another problem is that there may be other time course contingencies that are not global to local in nature. Several possible mechanisms have been discussed, and two other examples are given later. A more elegant explanation would involve principles that are more general than global and local. One possibility is the constituency model.

\section{The Constituency Model}

The constituency model is a framework for conceptualizing time course contingencies that is more general than the global-to-local model but more explicit than the contingency hypothesis. Two potentially important properties of the model are that it relates attentional resources to perception and it accounts for the present effects in terms of properties of evolving percepts, without recourse to strong top-down mechanisms.
In the model, feature information is integrated by local observers that report on a number of stimulus dimensions. For simplicity, I shall assume that the observers report only on the location and orientation of the lines nearest to their location. Thus, an observer in a given location might report "a vertical line passing through my location" or "a vertical line far (or near) to the left of me." Observers may also report on multiple lines (e.g., "a vertical and a horizontal line"). Initially, observers' reports are approximate.

Reports are interpreted at the blackboard level, in terms of more general hypotheses. At the blackboard level, hypotheses are (a) generated, (b) related to each other or combined (this serves to bind together features), and (c) verified through the gathering of increasingly detailed reports. However, processing resources are needed for these tasks. The amount of resources allocated to a given hypothesis is a direct function of the inclusiveness of the hypothesis, that is, the number of reports it incorporates. Thus, if a few reports imply a hypothesis, enough resources will be allocated to generate the hypothesis and possibly relate it to other reports or hypotheses. If other reports or hypotheses are consistent with the hypothesis, it will become more inclusive and will be allocated additional resources. As a hypothesis's strength increases (through support and interrelations), the additional resources are used to gather increasingly detailed information from the observer level and from relations at the blackboard level. This creates a circuit in which the strength of a hypothesis, the resources it receives, and its eventual degree of integration and resolution are contingent on a continuing match between the hypothesis and the accumulating visual evidence. Of course, if the hypothesis does not match new information, it will begin to lose its constituency and its processing resources.

In this model, primes that contain consistent global information would be effective early in processing because they would activate global, inclusive hypotheses. When more detailed information is available later in processing, hypotheses and processing resources would be available to interpret that information efficiently. Also, if the first stimulus is a target, then a global representation should be established from it. (Distinctive local features will also be extracted, but less efficiently than later in processing). If the target is followed by a local prime, the local information should be readily interpreted, producing a large facilitation effect. If the target is followed by a global prime, much of the global information would not be useful; however, the detailed outline of the prime could contribute some distinctive features, producing a facilitation effect that is smaller than that of late local primes.

In contrast to early global information, early local information would activate less inclusive, nonglobal hypotheses. The hypotheses would not be as easily interrelated and would not attract large amounts of processing resources, although they may be useful insofar as they contain distinctive feature information. Because the hypotheses are less inclusive, later information (from the target) would not be processed as efficiently as in the case of early global information.

The effectiveness of imprecise primes in Experiments 1-3 can be explained by a change over time in the resolution of 
spatial information. Early in processing, the spatial information in the observer's reports and the hypotheses would be coarse. Only after processing resources have been attracted (by inclusive hypotheses) would the spatial information become fine grained in nature.

The constituency model can also explain the inhibitory effects of inconsistent primes and begin to explain the lack of inhibitory effects of simple patterns (Sanocki, 1991a). Inconsistent object primes would cause the construction of inappropriate global hypotheses; these hypotheses would attract resources, depleting the resources available for appropriate hypotheses implied by the target stimulus. Such effects may be more pronounced with objects than with simple patterns because objects are more complicated and thus would cause more complex, resource-intensive hypotheses to be generated. With patterns, simpler hypotheses would be generated and therefore more resources would remain for prime-inconsistent hypotheses. Thus, the inhibitory effects of patterns would be smaller. ${ }^{6}$

\section{Time Course Contingencies in Other Contexts}

To further develop the idea of time course contingencies, I shall briefly discuss some examples of contingencies contained in models developed for other domains. To account for the identification of objects rotated in orientation or depth, UlIman (1989) proposed a two-stage alignment model. In the first stage, the size and orientation of an object are normalized with respect to internal models in a sophisticated normalization process. In the second stage, the internal models are matched against the image. Because only one viewpoint instantiation of each model need be considered, the computational complexity of the matching process is greatly reduced. This is a time course contingency because the viewpoint matched in the second stage is dependent on the results of the earlier stage.

A second example is the activation verification model of word perception (Becker, 1980; Paap, Newsome, MacDonald, \& Schvaneveldt, 1982). In this model, when a word is presented it activates letter units and word units in a bottom-up manner. Word units activated beyond a threshold become part of a candidate set and are matched against stimulus information in a top-down verification process. Because top-down processing is limited to candidates, processing demands are limited, another example of a contingency.

When the model is applied to effects of semantic context (e.g., Becker, 1980; Schvaneveldt \& MacDonald, 1981), it is assumed that a set of candidates is activated on the basis of semantic features of the context. In this assumption, the model differs from the present idea of a contingency. Although context dependence is very much a part of the present idea, a purely semantic-based dependence is stronger than the present idea because it does not involve stimulus information. An alternative approach to context effects that is more in line with the present ideas is that semantic information is used in the processing of candidate words selected on the basis of bottom-up information (e.g., Sanocki et al., 1985).
One final comment concerns a parallel between the constituency model's and the activation verification model's explanations of the relative sizes of inhibitory and facilitory effects. Becker (1980) noted that semantic priming experiments can yield either of two general patterns: When primetarget relations are strong, the facilitation effects of related primes are large and the inhibitory effects of unrelated primes are small, relative to neutral conditions (facilitation dominance). In contrast, when primes are weakly related to many possible targets, their facilitation effects are small and the inhibition effects of unrelated primes are large (inhibition dominance). According to Becker, these differing patterns are caused by differences in the number of candidates activated by the prime. Similarly, in the constituency model, such effects can be explained in terms of the number of hypotheses activated. Highly constraining primes would generate a few hypotheses, whereas more general primes would generate many hypotheses. Resource consumption could vary with the number of hypotheses, meaning that with many hypotheses resources would be more depleted and overall performance lower relative to neutral conditions. This would reduce performance in both prime conditions relative to neutral conditions, producing inhibition dominance.

Although these models further illustrate possible time course contingencies, the success or failure of the general idea should not be tied to any one model, because time course contingencies may take a number of different forms.

\section{Conclusion}

The results of the present experiments support the claim of the contingency hypothesis that the importance of different types of information changes over the time course of object identification, with early information being used to increase the efficiency of later processing. The results are consistent with the specific types of contingencies predicted by the global-to-local model, although other types of contingencies may also be important. The contingency hypothesis is an alternative to strictly bottom-up models and may provide a fruitful way of understanding how the perceptual system deals with the computational burdens of object identification.

\footnotetext{
${ }^{6}$ Sanocki (1991a) obtained no evidence of (even small) inhibitory effects. However, as noted in that article, inhibitory effects may have been underestimated slightly because the neutral condition used (a no-prime condition) did not signal the exact onset of the target, whereas the prime conditions did signal exact onset.
}

\section{References}

Becker, C. A. (1980). Semantic context effects in visual word recognition: An analysis of semantic strategies. Memory \& Cognition, 6, 493-512.

Biederman, 1. (1972). Human performance in contingent information-processing tasks. Joumal of Experimental Psychology, 93, 219-236.

Biederman, I. (1987). Recognition-by-components: A theory of human image understanding. Psychological Review, 94, 115-147. 
Biederman, I., Blickle, T. W., Teitelbaum, R. C., Klatsky, G. J., \& Mezzanotte, R. J. (1988). Object identification in multi-object, non-scene displays. Journal of Experimental Psychology: Learning, Memory, and Cognition, 14, 456-467.

Biederman, I., Teitelbaum, R. C., \& Mezzanotte, R. J. (1983). Scene perception: A failure to find a benefit from prior expectancy or familiarity. Journal of Experimental Psychology: Learning, Memory, and Cognition, 9, 411-429.

Boer, L. C., \& Keuss, P. J. G. (1982). Global precedence as a postperceptual effect: An analysis of speed-accuracy tradeoff functions. Perception \& Psychophysics, 31, 358-366.

Bouma, H. (1971). Visual recognition of isolated lower-case letters. Vision Research, 11, 459-474.

Breitmeyer, B. G. (1984). Visual masking: An integrative approach. New York: Oxford University Press.

Broadbent, D. E. (1977). The hidden preattentive process. American Psychologist, 32, 109-118.

Di Lollo, V. (1980). Temporal integration in visual memory. Joumal of Experimental Psychology: General, 109, 75-97.

Earhard, B. (1990). The generality of outside-in processing routines in the analysis of form. Canadian Journal of Psychology, 44, 14-29.

Earhard, B., \& Walker, H. (1985). An "outside-in" processing strategy in the perception of form. Perception \& Psychophysics, 38, 249-260.

Eriksen, C. W., \& Collins, J. F. (1967). Some temporal characteristics of visual pattern perception. Journal of Experimental Psychology, 74, 476-484.

Eriksen, C. W., O’Hara, W. P., \& Eriksen, B. A. (1982). Response competition effects in same-different judgments. Perception \& Psychophysics, 32, 261-270.

Estes, W. K. (1978). Perceptual processing in letter recognition and reading. In E. C. Carterette \& M. P. Friedman (Eds.), Perceptual processing (pp. 163-220). San Diego, CA: Academic Press.

Garner, W. R. (1985). Contingent information processing: Contingent and precued classification. Perception \& Psychophysics, 38, 237-248.

Garner, W. R. (1987). Location and color as cuing dimensions in contingent classification. Perception \& Psychophysics, 41, 202-210.

Graham, N. V. S. (1989). Visual pattern analyzers. New York: Oxford University Press.

Henderson, L. (1977). Word recognition. In N. S. Sutherland (Ed.), Tutorial essays in experimental psychology (Vol. 1). Hillsdale, NJ: Erlbaum.

Hummel, J. E., \& Biederman, I. (1992). Dynamic binding in a neural network for shape recognition. Psychological Review, 99 , 480-517.

Humphreys, G. W., \& Quinlan, P. T. (1988). Priming effects between two-dimensional shapes. Journal of Experimental Psychology: Human Perception and Performance, 14, 203-220.

Inside Macintosh. (1985). Reading, MA: Addison-Wesley.

Jacoby, L. L., Baker, J., \& Brooks, L. (1989). Episodic effects on picture identification: Implications for theories of concept learning and theories of memory. Journal of Experimental Psychology: Learning, Memory, and Cognition, 15, 275-281.

Johnston, J. C. (1978). A test of the sophisticated guessing theory of word perception. Cognitive Psychology, 10, 123-153.

Johnston, J. C., \& McClelland, J. L. (1973). Visual factors in word perception. Perception \& Psychophysics, 14, 365-370.

Jolicoeur, P. (1985). The time to name disoriented natural objects. Memory \& Cognition, 13, 289-303.

Keren, G., \& Baggen, S. (1981). Recognition models of alphanumeric characters. Perception \& Psychophysics, 29, 234-246.

Kimchi, R., \& Palmer, S. (1985). Separability and integrality of global and local levels of hierarchical patterns. Journal of Experimental Psychology: Human Perception and Performance, 11, 673-688.

Kinchla, R. A., \& Wolfe, J. M. (1979). The order of visual processing: "Top-down," "bottom-up" or "middle-out." Perception \& Psychophysics, 25, 225-231.

Krueger, L. E., \& Chignell, M. H. (1985). Same-different judgments under high speed stress: Missing feature principle predominates in early processing. Perception \& Psychophysics, 38, 188-193.

Krumhansl, C. L. (1982). Abrupt changes in visual stimulation enchance processing of form and location information. Perception \& Psychophysics, 32, 511-523.

Luce, R. D. (1959). Individual choice behavior. New York: Wiley. Lupker, S. J. (1979). On the nature of perceptual information during letter perception. Perception \& Psychophysics, 25, 303-312.

Massaro, D. W. (1989). Testing between the TRACE model and the fuzzy logical model of speech perception. Cognitive Psychology, 21, 398-421.

Massaro, D. W., \& Friedman, D. (1990). Models of integration given multiple sources of information. Psychological Review, 97, 225-252.

Massaro, D. W., \& Sanocki, T. (in press). Visual information processing in reading. In D. Willows, R. Kruck, \& E. Corcos (Eds.), Visual processes in reading and reading disabilities. Hillsdale NJ: Erlbaum.

McClelland, J. L. (1978). Perception and masking of wholes and parts. Journal of Experimental Psychology: Human Perception and Performance, 4, 210-223.

McClelland, J. L. (1985). Putting knowledge in its place: A scheme for programming parallel processing structures on the fly. Cognitive Science, 9, 113-146.

McClelland, J. L. (1991). Stochastic interactive processes and the effects of context on perception. Cognitive Psychology, 23, 1-44.

McClelland, J. L., \& Miller, J. (1979). Structural factors in figure perception. Perception \& Psychophysics, 26, 221-229.

McClelland, J. L., \& Rumelhart, D. E. (1981). An interactive model of context effects in letter perception: Part 1. An account of basic findings. Psychological Review, 80, 375-407.

Miller, J. (1981). Global precedence in attention and decision. Journal of Experimental Psychology: Human Perception and Performance, 9, 1161-1174.

Miller, J. (1989). The control of attention by abrupt visual onsets and offsets. Perception \& Psychophysics, 45, 567-571.

Navon, D. (1977). Forest before trees: The precedence of global features in visual perception. Cognitive Psychology, 9, 353-383.

Navon, D. (1981). The forest revisited: More on global precedence. Psychological Research, 43, 1-32.

Navon, D. (1991). Testing a queue hypothesis for the processing of global and local information. Journal of Experimental Psychology: General, 120, 173-189.

Neisser, U. (1967). Cognitive psychology. Englewood Cliffs, NJ: Prentice Hall.

Neisser, U. (1976). Cognition and reality. San Francisco: Freeman. Norman, D. A., \& Bobrow, D. G. (1976). On the role of active memory processes in perception and cognition. In C. N. Cofer (Ed.), The structure of human memory (pp. 114-132). San Francisco: Freeman.

Oden, G. C. (1979). A fuzzy logical model of letter identification. Journal of Experimental Psychology: Human Perception and Performance, 5, 336-352.

Paap, K. R., Newsome, S. L., McDonald, J. E., \& Schvaneveldt, R. W. (1982). An activation-verification model for letter and word recognition: The word-superiority effect. Psychological Review, 89, 573-594. 
Paap, K. R., \& Ogden, W. C. (1981). Letter encoding is an obligatory but not capacity-demanding operation. Journal of Experimental Psychology: Human Perception and Performance, 7, 518-527.

Palmer, S. E. (1975). Visual perception and world knowledge: Notes on a model of sensory-cognitive interaction. In D. A. Norman, D. E. Rumelhart, \& the LNR Research Group, Explorations in cognition (pp. 279-307). San Francisco: Freeman.

Palmer, S. E. (1977). Hierarchical structure in perceptual representation. Cognitive Psychology, 9, 441-474.

Palmer, S. E., Rosch, E., \& Chase, P. (1981). Canonical perspective and the perception of objects. Attention and performance IX (pp. 135-151). Hillsdale, NJ: Erlbaum.

Pomerantz, J. R. (1983). Global and local precedence: Selective attention in form and motion perception. Journal of Experimental Psychology: General, 112, 516-540.

Posner, M. I. (1980). The orienting of attention. Quarterly Journal of Experimental Psychology, 32, 3-25.

Price, C. J., \& Humphreys, G. W. (1989). The effects of surface detail on object categorization and naming. Quarterly Journal of Experimental Psychology, 4IA, 797-828.

Rumelhart, D. E. (1977). Toward an interactive model of reading. In S. Dornic (Ed.), Attention and performance IV. Hillsdale, NJ: Erlbaum.

Sanocki, T. (1987). Visual knowledge underlying letter perception: Font-specific, schematic tuning. Journal of Experimental Psychology: Human Perception and Performance, 13, 267-278.

Sanocki, T. (1988). Font regularity constraints on the process of letter recognition. Journal of Experimental Psychology: Human Perception and Performance, 14, 472-480.

Sanocki, T. (1990). Effects of font-and letter-specific experience on letter recognition. Unpublished manuscript.

Sanocki, T. (1991a). Effects of early common features on form recognition. Perception \& Psychophysics, 50, 490-497.

Sanocki, T. (1991b). Intra- and inter-pattern relations in letter recognition. Journal of Experimental Psychology: Human Perception and Performance, 17, 924-941.

Sanocki, T., Goldman, K., Waltz, J., Cook, C., Epstein, W., \& Oden,
G. C. (1985). Interaction of stimulus and contextual information during reading: Identifying words in sentences. Memory \& Cog. nition, 13, 145-157.

Sanocki, T., \& Oden, G. C. (1991). Perceptual adjustments on representations of familiar patterns: Change over time and relational features. Perception \& Psychophysics, 50, 28-44.

Schvaneveldt, R. W., \& McDonald, J. E. (1981). Semantic context and the encoding of words: Evidence for two modes of stimulus analysis. Journal of Experimental Psychology: Human Perception and Performance, 7, 673-687.

Shibuya, H., \& Bundesen, C. (1988). Visual selection from multielement displays: Measuring and modeling effects of exposure duration. Journal of Experimental Psychology: Human Perception and Performance, 14, 591-600.

Townsend, J. T. (1981). Some characteristics of visual whole report behavior. Acta Psychologica, 47, 149-173.

Townsend, J. T., \& Ashby, F. G. (1982). Experimental test of contemporary mathematical models of visual letter recognition. Journal of Experimental Psychology: Human Perception and Performance, 8, 834-864.

Townsend, J. T., Hu, G. G., \& Kadlec, H. (1988). Feature sensitivity, bias, and interdependencies as a function of energy and payoffs. Perception \& Psychophysics, 43, 575-592.

Tversky, A. (1977). Features of similarity. Psychological Review, $84,327-352$.

Ullman, S. (1989). Aligning pictorial descriptions: An approach to object recognition. Cognition, 32, 193-254.

Waltz, D. (1975). Understanding line drawings of scenes with shadows. In P. H. Winston (Ed.), The psychology of computer vision (pp. 19-92). New York: McGraw-Hill.

Yantis, S., \& Jonides, J. (1984). Abrupt visual onsets and selective attention: Evidence from visual search. Journal of Experimental Psychology Human Perception and Peformance, 10, 601-621.

Received February 18, 1992

Revision received October 14, 1992 Accepted October 20, 1992 